\title{
Coupled experimental and computational investigation of omega phase evolution in a high misfit titanium-vanadium alloy
}

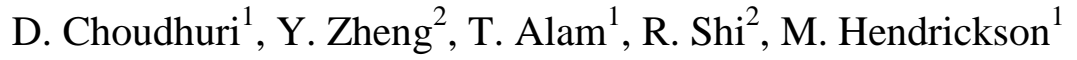

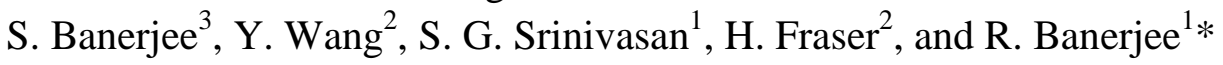 \\ ${ }^{1}$ Department of Materials Science and Engineering, University of North Texas, Denton, \\ TX, 76191, USA \\ ${ }^{2}$ Department of Materials Science and Engineering, The Ohio State University, \\ Columbus, OH 4310, USA \\ ${ }^{3}$ Bhabha Atomic Research Centre, Mumbai, India \\ * Corresponding author: Raj.Banerjee@unt.edu
}

\begin{abstract}
Morphological and compositional evolution of omega $(\omega)$ precipitates in a model Titanium-20wt\%Vanadium (or 19at.\%V) alloy has been systematically investigated by coupling transmission electron microscopy and atom probe tomography with atomistic $a b$ initio and continuum microelasticity computations. The initial water quenched microstructure comprised of a fine scale distribution of athermal $\omega$ precipitates, which form congruently from the $\beta$ phase via a complete displacive collapse of $\{222\}_{\beta}$ planes, that has been rationalized based on DFT computations. Subsequent annealing at $300^{\circ} \mathrm{C}$, over progressively increasing time periods, resulted in isothermal evolution of the $\omega$ precipitates, whose morphology changes from ellipsoidal to cuboidal, accompanied with $\mathrm{V}$ rejection. The highly $\mathrm{V}$-enriched $\beta$ matrix consisted of short $\mathrm{V}-\mathrm{V}$ bond lengths, further distorting the $b c c$ lattice, and increasing the $\beta / \omega$ misfit. This facilitates the change in the morphology of omega precipitates from ellipsoidal to cuboidal resulting in a $\{001\}_{\beta}$ habit plane for these precipitates. The coupled experimental and computational approach
\end{abstract}


permits rationalizing the evolution of $\omega$ precipitate morphology and composition in such high $\beta-\omega$ misfit $\beta$-Ti alloys. 


\section{Introduction}

Omega $(\omega)$ phase formation is an interesting case of displacive or coupled displacivediffusional transformation found in Group IV metals (e.g. Ti, Zr) and their alloys [1-7].

The past six decades has witnessed substantial amount of research effort to understand $\omega$ precipitation and its influence on the mechanical and superconducting properties [6]. In recent years, $\omega$ formation has gained renewed attention due to its role as a precursor to fine scale $\alpha$ phase precipitation in Ti-alloys [7-15], which results in higher strengths $[16,17]$. In pure Ti $\omega$ forms only under the influence of large compressive stress/pressures during hydrostatic or shock compression [1-5]. In contrast $\omega$ forms at ambient pressure only in bcc ( $\beta$ ) Ti alloys containing $\beta$-stabilizing elements like $\mathrm{Mo}, \mathrm{V}, \mathrm{Nb}$ etc [6].

In $\beta$-stabilized Ti alloys, $\omega$ forms athermally by quenching form the $\beta$ phase field (at high temperature), to a much lower temperature e,g, room temperature [6]. Athermal $\omega$ formation is typically with associated with an atomic shuffle mechanism [18-29], which, according to De Fontaine, can also be described as either $\frac{2}{3}<111>$ longitudinal or $\frac{1}{3}<112>$ transverse displacement wave in the bcc lattice $[19,23]$. The presence of this displacement wave was latter confirmed via neutron diffraction experiments, which showed $\frac{2}{3}<111>$ wave vector in the phonon spectra [30]. However, contrary to experimental observations, De Fontaine's harmonic lattice theory based mechanism also implied that $\beta \rightarrow \omega$ transformation will reach completion [28]. Latter, Cook modified this mechanism by introducing anharmonicity to the lattice free energy, and expressed this energy as a Landau polynomial $\mathrm{F}=A \eta^{2}+B \eta^{3}+C \eta^{4}+\ldots$, where the constants $\mathrm{A}$ and $\mathrm{C}$ are positive definite and B is negative, and $\eta$ planar displacement [24-26]. Crucially, the modified theory indicated an energy barrier leading to $\omega$ formation [24-27], which was 
recently confirmed via first principle calculations involving $\beta \rightarrow \omega$ transformation in TiMo alloys [31]. From an experimental point of view, these theoretical insights suggest that $\beta \rightarrow \omega$ transformation is due to the collapse of neighboring $\{222\}_{\beta}$ planes, and several microscopy-based investigations have been carried out to observe this planar collapse.

Sukedai et.al. carried out one of the first high-resolution imaging of $\omega$ in quenched Ti-11at.\%Mo and Ti-14at.\%Mo alloys by coupling high-resolution transmission electron microscopy (HRTEM) and image simulations [32-34]. Recently, Devaraj et.al. examined the $\omega$ structure in Ti-9at.\%Mo in unprecedented detail by using aberration corrected (Cs corrector in condenser lens $[35,36])$ high resolution scanning transmission electron microscopy (HRSTEM) fitted with high angle dark field (HAADF) detector $[37,38]$. The HAADF-STEM methodology allows one to image atomic columns with sub-atomic resolution, while simultaneously showing the differences in the average atomic number of such columns via contrast differences [31,35-37]. However, these atomically resolved examinations of the $\omega$ structure was carried out in only low misfit alloys exhibiting small difference in atomic radii of $\mathrm{Ti}$ and the solute atoms such as Mo atoms.

One of the earliest study on as-quenched high misfit Ti-V alloys was performed by McCabe et.al. [20]. Their observations indicated that $\omega$ structure consist of fully collapsed $\{222\}_{\beta}$ planes, corresponding to "ideal" $\omega$ structure (symmetry P6/mmm), even at very high levels of solute concentration e.g 19 at.\%V compared to Ti-Mo alloys [20]. They deduced the presence of an ideal $\omega$ structure from selected area electron diffraction patterns (SADPs) recorded along $[011]_{\beta}$ zone axis [7,20-22]. Furthermore, higher alloy 
compositions (25 - 33at.\%) produced pronounced reciprocal lattice streaking (RLS) suggesting the presence of partially collapsed $\{222\}_{\beta}$, while very faint RLS was noted at concentrations in excess of 33at.\%V [20]. Regardless, a conclusive experimental evidence of full / partial $\{222\}_{\beta}$ collapse of athermal $\omega$ in quenched Ti-V alloys is yet to be presented [18-38]. More importantly, it is unclear why athermal $\omega$ is still observed at such high levels of solute concentration in Ti-V alloys, and not in Ti-Mo with comparable levels of solute atoms. Nevertheless, we believe that the displacement wave mechanism is likely responsible for the athermal $\omega$ formation in both alloys. However, displacement mechanism[19,24-26,30], or other recent theoretical works [5,31,39-43] offer little physical insight into the formation of athermal or isothermal $\omega$ at high/low levels of solute concentrations in beta-stabilized $\omega$-forming Ti-alloys.

The differences in high and low misfit $\beta$-Ti alloys become even more prominent when they are exposed to annealing below their $\omega$-solvus temperatures [7, 18-29]. Such long-term annealing results in fully developed $\omega$ precipitates, called isothermal $\omega$ ), that are rich in $\mathrm{Ti}$ and lean on solute elements [7, 18-29]. While, isothermal $\omega$ in low misfit systems retain ellipsoidal morphology, but in high misfit alloys they eventually acquire cuboidal morphology [7, 18-29]. The formation of isothermal $\omega$ for low misfit systems has been well established via a model Ti-Mo alloy [7, 18-29, 37]. By coupling structural and compositional observations obtained through HRSTEM and Atom Probe Tomography (APT) respectively, Devaraj et.al. demonstrated that isothermal $\omega$ precipitates form in a compositionally phase-separated $\beta$-matrix $[37,38]$. In contrast, a conclusive mechanism for the nucleation and growth of isothermal $\omega$ is yet to be resolved in case of $\mathrm{Ti}-\mathrm{V}$ system. Using conventional TEM of Ti-50at.\% $\mathrm{V}$ and thermodynamic 
calculations, Koul et.al. suggested that compositional fluctuations in the $\beta$ phase cannot be associated with the formation of isothermal $\omega$ [44]. Furthermore, the role of prior athermal $\omega$ on isothermal $\omega$ formation cannot be deduced from their work [44]. In a latter study on Ti-10V-2Fe-3Al (wt\%) or Ti-9.3V-1.7Fe-5Al (at\%), Duerig et.al. proposed that compositional fluctuations near the "quasi-static" athermal $\omega$ particles plays a role in the formation of isothermal $\omega$ precipitates $[27,28]$. Their proposition was primarily based on microstructures observed via dark-field TEM imaging [27, 28], and not by measuring the composition of $\omega$ and $\beta$ phases at different stages of annealing. Recently, Ng et.al. reported compositional fluctuations preceding isothermal $\omega$ formation during a slow continuous cooling of a Ti-9.6V-4.6Cu (at\%) alloy [45]. However, isothermal $\omega$ stability regime / or temperature is rather difficult to establish because of the continuous cooling rate employed in this work [45].

A systematic understanding of compositional evolution in Ti-V alloys during the formation and coarsening of $\omega$ will allow us to understand the role of misfit strains on morphological evolution of isothermal $\omega$ from ellipsoids (oriented along $\langle 111\rangle_{\beta}[7,19]$ ) to the final cuboidal shape (with faces parallel to $(001)_{\beta}[7,27,28]$. It is expected that misfit strains, due to changing $\omega$ and $\beta$ lattice parameters during annealing may play a key role in the observed change in morphology $[7,27,28]$. In fact the dependence of $\omega$ and $\beta$ lattice parameters on $V$ concentration was demonstrated by Aureloi et.al. by using neutron diffraction [46]. These extensive results mandated coupling lattice and compositional information (from e.g APT $[37,38,46]$ ) to reexamine the role of $\mathrm{V}$ concentration on the isothermal $\omega$ morphology. 
The present study is a systematic investigation of athermal and isothermal $\omega$ precipitation in a model Ti-20wt.\%V or Ti-19at.\%V alloy, by water quenching and subsequent isothermal annealing to $256 \mathrm{Hrs}$. Ti-19at.\%V was chosen because it forms $\omega$ in the quenched-in condition [20] and, reportedly, phase separates upon isothermal annealing [27, 28]. Microstructural characterization was carried out by coupling state-ofthe-art aberration corrected HAADF-STEM microscopy, conventional TEM, and APT. The experimental results were complimented by density functional theory (DFT)-based first principle calculations, and microelasticity theory based strain energy calculations [48]. By combining the experimental observations with the computational results, we addressed the following salient aspects of $\omega$ precipitation in high $\beta-\omega$ misfit alloys:

1. The degree of $\{222\}_{\beta}$ collapse and the composition of $\omega$ precipitates in the asquenched condition.

2. Examined systematically $\omega$ morphology and volume fraction, and solute partitioning between $\omega$ and $\beta$ during isothermal annealing.

3. Established electronic and ground state structures of $\omega$ and $\beta$ phases for relevant solute concentrations.

4. Characterized the effect of solute partitioning between $\omega$ and $\beta$ on the habit plane of the $\omega$ precipitates.

\section{Experimental and Computational Methods}

Vacuum arc melted Ti-20wt.\%V or Ti-19at.\%V samples were obtained from Air Force Research Laboratory, Dayton, OH. The alloy ingots were produced by arc-melting Ti sponge and $\mathrm{V}$ pellets mixed in appropriate proportions inside a vacuum furnace. For heat 
treatments, the samples were first cut into $3 \times 2 \times 10 \mathrm{~mm}$ coupons, then wrapped in Ti foils along with small pieces of sponge Ti. The wrapped specimens were beta solutionized at $900^{\circ} \mathrm{C}$ for 1 hour in a tube furnace with flowing Argon atmosphere and followed by water quenching to room temperature. The water quenched coupons were subsequently annealed at $300^{\circ} \mathrm{C}$ for 1,64 and $256 \mathrm{~h}$; followed by water quenching. For annealing experiments, each sample was wrapped in the same procedure described above. To probe the early stages of $\omega$ precipitation, cylindrically cut $\beta$-solutionized specimens were annealed for 1 and 10 mins at $300^{\circ} \mathrm{C}$ inside a LINSEIS RITA L78 Quenching dilatometer maintained in He environment. The dilatometer allowed a strict control, via computerhardware interface, over the annealing time and heating/cooling rates (in this case $\left.100^{\circ} \mathrm{C} / \mathrm{min}\right)$.

TEM foils for microstructural characterization were prepared by using both focused ion beam milling (FEI Nova NanoLab 20system) and via conventional methods (dimple grinding and io-milling). Atomic resolution HAADF-STEM imaging was carried out with FEI TITAN ${ }^{\circledR} 80-300$ microscope operating at $300 \mathrm{kV}$, and equipped with a CEOS proper aberration corrector; experimental parameters used in this work is described elsewhere [37]. Conventional TEM microscopy was performed with a FEI Tecnai $\mathrm{G}^{2} \mathrm{TF} 20^{\mathrm{TM}}$ operating at $200 \mathrm{kV}$. For atom probe tomography (APT) investigation, conical tips were extracted from heat-treated specimens via FIB milling, and these tips were exposed to pulsating voltage inside the chamber of a local electrode atom probe (LEAP 3000x HR®). Details of the APT experiments and the corresponding data analysis are provided elsewhere [49]. 
First principles calculations were carried out with the Vienna Ab-initio Simulation Package (VASP) employing the projector augmented plane-wave (PAW) method, with electron exchange and correlation described by the generalized gradient approximation (GGA) [50-53]. Convergence tests carried out on multiple supercells sites (containing both $\mathrm{Ti}$ and $\mathrm{V}$ ) indicated that the a cut-off energy of $420 \mathrm{eV}, 0.18 \AA^{-1} \mathrm{k}$-point spacing and $0.2 \mathrm{eV}$ Methfessel-Paxton smearing width was sufficient for our calculations. All calculations was performed at $0 \mathrm{~K}$ using Brillouin zone integration at $10^{-7} \mathrm{eV}$ convergence threshold for self-consistent calculations and $\sim 1 \mathrm{meV} /$ atom for global energy convergence.

\section{Results}

\subsection{Experimental Results}

Experimental results acquired after water quenching and subsequent annealing Ti19at.\% V alloy will be presented in this section.

\subsubsection{Omega precipitation after water quenching: Initial Condition}

Phase identification and the associated structural features of the precipitate phase were examined via conventional and atomic resolution microscopy; as shown in Figure 1. A selected area diffraction pattern (SADP) recorded along [011] $\beta$ zone axis (ZA), in Figure 1(a), indicated the presence of distinct additional reflections that can attributed to the $\omega$ phase (along with the primary $\beta$ reflections), with the intensity maximums from the $\omega$ reflections lying at the $1 / 3$ and $2 / 3\langle 211\rangle_{\beta}{ }^{*}$ (marked with an arrow, and $*=$ reciprocal space vector). The $\omega / \beta$ orientation relationship (OR) derived from the [011] $\beta$ SADP and 
other zone axes was, $(011)_{\beta}\left\|(2 \overline{1} \overline{1} 0)_{\omega},[\overline{2} 2 \overline{2}]_{\beta}\right\|[0001]_{\omega}$ and $[2 \overline{1} \overline{1}]_{\beta} \|[0 \overline{1} 10]_{\omega}$, which is also schematically shown with plane motifs of $(2 \overline{1} \overline{1} 0)$ of an $\omega$ variant and $(011)_{\beta}$ in Figure 1(a). The additional faint reflections (e.g along $[011]_{\beta}{ }^{*}$ and $[\overline{2} 00]_{\beta}{ }^{*}$ ) are double diffractions [7,20], and are indicated with squares. It may be noted that, the positions of $\omega$ intensity maximas in the (011) $\beta$ SADP suggest an "ideal" $\omega$ crystal structure (space group P6/mmm), which, based on past studies [7, 18-30], consists of fully collapsed $\{222\}_{\beta}$ planes.

To further clarify the $\omega$ structure in the water quenched condition, HAADFSTEM (hereon HRSTEM) examination was carried out with an aberration corrected TEM. Figure 1(b) shows a relatively low magnification, atomically resolved, and background corrected HRSTEM image recorded along [011 $]_{\beta}$ ZA, which depicts a small ellipsoidal $\omega$ precipitate $(\sim 7 \mathrm{~nm}$ long and $\sim 2 \mathrm{~nm}$ wide). Figures 1 (c) and 1 (d) show the atomic arrangement within the $\omega$ and parent $\beta$ phases in enlarged views of similar magnification. Comparing the two figures we note that the quenched-in athermal $\omega$, indicated with a red rectangular motif, consists of fully collapsed $\{222\}_{\beta}$ planes. In other words HRSTEM results confirms that the structure of $\omega$ phase formed after water quenching is akin to "ideal" $\omega$ crystal structure - consistent with the SADP presented in Figure 1(a).

APT was utilized to examine the composition of $\omega$ precipitates in the waterquenched specimens. Figure 2 shows the APT results obtained from a representative dataset. Figure 2(a) shows a 3D reconstruction of an APT tip using red colored V ions. A visual examination of the reconstructed volume did not indicated any visible signature of solute partitioning, therefore compositional analysis was carried out with binomial 
analysis (Figure 2(b)) and 1D profiling (Figure 2(c)-(d)) of the APT tip. The binomial analysis is a statistical algorithm (implement in IVAS 3.6.6®), which measures the composition by dividing the entire 3D volume into blocks of atoms (in this case 100 atoms), and $\mathrm{V}$ concentration in each block is plotted as a frequency distribution (Figure 2(b)) [47]. A total of 8000 of such blocks was used in the present analysis. The data points in Figure 2(b), indicated as "observed", shows the results from such an analysis. Also overlaid in dotted line is the distribution expected from a uniform solid solution [47]. Figure 2(b) shows that the data points closely follow the dotted line - meaning that alloy composition is uniform after water quenching, representative of a random solid solution. Further, 1D compositional analysis was carried out by placing a cylinder (diameter $\sim 5 \mathrm{~nm}$ ) along the axis of the APT tip (Figure 2(c)) and calculating V concentration along the length of the cylinder. Figure 2(d) indicates that sampled alloy composition is statistically uniform along the tip axis, since the fluctuations are within the error bars. The measured average alloy composition $(21.9 \pm 4.5$ at.\%V) from 1D profiling was close to the bulk composition ( 19at.\%) within an error margin. The APT analysis also inidcated the following impurity levels in the solutionized alloy: 1.8at.\%O, 0.14at.\% $\mathrm{Si}$ and 0.24at.\%Ti. Notwithstanding, the APT results did not reveal any discernable solute (i.e vanadium) partitioning or compositional fluctuations in the waterquenched alloy - consistent with literature reports [18-30, 37, 38].

Thus, the coupled TEM and APT investigation revealed that water quenching of Ti-19at.Vresults in the precipitation of fine scale ellipsoidal $\omega$ precipitates with fully collapsed $\{222\}_{\beta}$ planes composition similar to the parent $\beta$ matrix. A rationale for this observation will be discussed in a later section. Nevertheless, based on these results it is 
likely that the "quenched-in" compositionally congruent omega precipitates are a result of an athermal transformation mechanism within the parent beta matrix.

\subsubsection{Evolution of Omega phase during isothermal annealing at $300^{\circ} \mathrm{C}$}

The morphological and compositional evolution of the quenched-in $\omega$ precipitates was further examined after isothermally annealing the water quenched (WQ) specimens at $300^{\circ} \mathrm{C}$ for short durations ( 1 and 10 mins), as well as longer durations $(1,64$ and $256 \mathrm{H})$.

\section{Morphological evolution}

The change in $\omega$ morphology with annealing time has been presented using a series of dark-field TEM (DFTEM) images, shown in Figure 3, while the insets show the corresponding $[011]_{\beta}$ SADPs and the $\omega / \beta$ OR via $[011]_{\beta}$ and $[2 \overline{1} \overline{1} 0]_{\omega}$ motifs. A close examination of the diffraction patterns from annealed specimens (top right insets in each dark-field micrographs) indicated that intensity maxima are present as the $1 / 3$ and $2 / 3$ $\langle 211\rangle_{\beta}{ }^{*}$ locations, which suggests that the structure of $\omega$ precipitates after annealing consists of fully collapsed $\{222\}_{\beta}$.

The DFTEM of isothermally annealed specimens indicated that between 1 mins to $1 \mathrm{~h}$ (Figures 3(b)-(c)) the $\omega$ precipitates grew (from an axial length of $\sim 8 \mathrm{~nm}$ to $\sim 15 \mathrm{~nm}$ ), and, interestingly, maintained a comparably high number density, comparable to the WQ condition (Figure 3(a). After $64 \mathrm{H}$ of annealing the $\omega$ precipitates coarsened (length along major axis $\sim 10 \mathrm{~nm}$ ) and exhibited morphologies ranging from ellipsoidal to nearcuboidal (Figure 3(e)). In comparison to the $1 \mathrm{Hr}$ microstructure (Figure 1(d), the 
number density of $64 \mathrm{H}$ annealed has noticeably reduced suggesting that coarsening was initiated between 1 and $64 \mathrm{H}$. These $\omega$ particles finally acquired cuboidal morphology (diagonal length $\sim 20 \mathrm{~nm}$ ) after long term annealing at $256 \mathrm{H}$ (Figure 3(f)) and the number density of the $\omega$ has not noticeably altered compared to the $64 \mathrm{H}$-annealed microstructure. The compositional evolution of these isothermal $\omega$ precipitates and the adjacent parent $\beta$ matrix after isothermal annealing at $300^{\circ} \mathrm{C}$, has been discussed below.

\section{Compositional Evolution}

APT samples were extracted from specimens annealed at $300^{\circ} \mathrm{C}$ for $10 \mathrm{~min}$ and for 1,64 and $256 \mathrm{H}$, and the datasets were analyzed by focusing on determining the compositions of $\omega$ and the adjacent $\beta$ matrix.

APT results from samples annealed for 10mins and $1 \mathrm{H}$ are shown in Figures 4(a) - (d) and Figure 4(e) respectively. Figure 4(a) shows a 3D atom probe reconstruction using $\mathrm{V}$ ions (red), and the $\omega$ precipitates delineated with 82 at.Visoconcentration surfaces (or isosurfaces $[47,54]$ ) in blue. The APT results show that 3D morphology and the number density of $\omega$ precipitates are comparable with the TEM observations (refer to Figure 3(c)). To quantify the $\mathrm{V}$ partitioning across the $\omega / \beta$ interface a proximity histogram (or proxigram [47, 54]) was calculated (Figure 4(b)) from the isosurfaces by only selecting those precipitates that lie completely inside the volume of the reconstructed tip (in Figure 4(a). The compositions of the $\omega$ and $\beta$ phase were determined to be $5.4 \pm 1.4$ at.Vand $23.4 \pm 0.5$ at.\%Vrespectively (see Table I). Solute distribution within the $\beta$ matrix is further investigated using $2 \mathrm{D}$ pseudo-colored $\mathrm{V}$ concentration maps in Figure 4(d). In the 2D map, the $\omega$ precipitates appear as solute 
depleted blue colored regions, while the color variations within the $\beta$ matrix is statistical due to compositional heterogeneities. A 1D composition profile (using a $3 \mathrm{~nm}$ diameter cylinder) of the $\beta$ matrix lying between two $\omega$ particles in Figure 4 (e) indicated V enrichment on average $\sim 25$ at $\%$ with peak values in excess of $30 \mathrm{at} \%$.

The reduction in $\mathrm{V}$ content in $\omega$ (from $\sim 21$ at.Vin $\mathrm{WQ}$ to $5.4 \mathrm{at} \% \mathrm{~V}$ ) in a matter of 10 mins or $600 \mathrm{sec}$ was rather surprising. Therefore, the subsequent compositional evolution of isothermal $\omega$ can conceivably occur in two ways: first, a gradual reduction in solute content as reported by Devaraj et.al.[37], or within 10mins of annealing the $\omega$ precipitates may have achieved an near equilibrium composition. To examine both possibilities APT was carried on a $1 \mathrm{Hr}$ annealed specimen (Figure 4(e)). Proxigram analysis of the $\omega$ precipitates indicated that compositions of the $\omega$ and $\beta$ phases were 5.8 \pm 1.4 and $22.7 \pm 1.0$ at. $\% \mathrm{~V}$. Thus, the statistically similar compositions of both $\omega$ and $\beta$ phases after 10 mins and $1 \mathrm{H}$ of annealing indicated that the isothermal $\omega$ precipitates achieve near equilibrium composition at very early stages of annealing at $300^{\circ} \mathrm{C}$.

Further, it should be pointed out that the compositions extracted from proxigrams are essentially an "average" composition in the reconstructed APT tip because we probe the composition across all interfaces -- as delineated by the isosurfaces $[47,54]$-- while 1D profiling samples a rather localized region. Furthermore, the "compositional fluctuations" spaced within $1 \mathrm{~nm}$ (line profile in Figure 4(d)), cannot be construed as a signature of phase separation, because they are a result of statistical noise overlaid on the measured compositions. Furthermore, a simple diffusivity calculation by using $2 \sqrt{ } \mathrm{Dt}$ (where $\mathrm{D}_{\mathrm{V} \text { in } \beta-\mathrm{Ti}}=1.68 \times 10^{-20} \mathrm{~m}^{2} / \mathrm{s}[55,56]$ and $\mathrm{t}=600 \mathrm{~s}$ ) indicates a diffusion distance of $\sim 6 \mathrm{~nm}$, which is much larger than the spatial separation of the "compositional 
fluctuations", but correlates rather well with the size scale of the $\omega$ precipitates. Therefore, APT results from 10mins annealed sample indicates that the composition evolution of the quenched-in $\omega$ precipitates occurs via the rejection of solute (in this case V) from the $\omega$ precipitates and a gradual solute enrichment in the adjoining $\beta$ regions.

APT results after 64 and $256 \mathrm{H}$ of annealing, presented in Figures 5 and 6 respectively, further supports the mechanistic insight obtained from examination of the 1 $\mathrm{Hr}$ annealed specimen. Figure 5(a) shows a high volume fraction of $\omega$ precipitates delineated with a 85at.\%Visosurface - and consistent with TEM dark-field observations. The corresponding proxigram in Figure $5(\mathrm{~b})$ indicates that composition of the $\omega$ precipitates $(5.3 \pm 0.6$ at $\% \mathrm{~V})$ remains comparable to that of the $1 \mathrm{Hr}$ annealed condition, while the composition of the adjoining $\beta$ matrix increased to $33.1 \pm 0.7$ at.V(increased by $\sim$ 10at\% compared to $1 \mathrm{Hr}$ of annealing). A 2D compositional map is shown in Figure 5(c) with the inset depicting the region of the APT tip chosen for mapping. A 1D composition profile across the $\beta$ rib separating two distinct isothermal $\omega$ precipitates, shown in Figure 5(d), indicated that the $\mathrm{V}$ concentration reached levels as high as 40at\%. After isothermal annealing at $300^{\circ} \mathrm{C}$ for $256 \mathrm{H}$, a high volume fraction of cuboidal $\omega$ precipitates was clearly observed in the APT reconstructions (Figure 6(a)). The proxigram (Figure 6(b)) indicated that the composition of the $\beta$ and $\omega$ were $35.0 \pm 0.7$ and $5.0 \pm 0.8$ at.Vrespectively. Furthermore, $\mathrm{V}$ enrichment between the $\omega$ precipitates increased to rather very high values ( $\sim 50$ at $\%$; a $\sim 18 \%$ increase from $1 \mathrm{H}$ condition) after $256 \mathrm{H}$ of annealing. The compositions of the $\omega$ and $\beta$ phases at different stages of isothermal annealing are summarized in Table I. 
Finally, by coupling the TEM and APT results we can summarize the key results obtained after isothermal annealing the Ti-20wt $\% \mathrm{~V}$ or Ti-19at.Valloy at $300^{\circ} \mathrm{C}$ :

1. At the early stages of annealing $(1 \mathrm{~min}$ to $1 \mathrm{H})$ the number density and volume fraction of isothermal $\omega$ precipitates appear to be comparable to athermal $\omega$ precipitates based on the dark-field images shown in Figures 3(a)-(d).

2. From the very early stages (10mins) of isothermal annealing, unlike the quenched-in $\omega$ precipitates, the isothermal ones exhibit a substantial difference in composition from the parent $\beta$ matrix (see Table I) and have a $\mathrm{V}$ content that is near equilibrium $(\sim 5$ at $\%)$.

3. Subsequent isothermal annealing at 64 and $256 \mathrm{H}$ did not result in any substantial change in solute content within the $\omega$ precipitates, while their morphology changed from spheroidal/ellipsoidal to cuboidal (Figure 3). The progressive growth of the $\omega$ precipitates (after 64 and $26 \mathrm{H}$ ) accompanied with an increase in the $\omega$ volume fraction resulted in a gradual increase in V solute content within the retained $\beta$ matrix (due to continued rejection of solute from the $\omega$ phase; depicted in Figures 4, 5, and 6), with local solute contents within the $\beta$ matrix reaching very high levels as compared to the average alloy composition e.g. $\sim 50$ at.Vafter $256 \mathrm{H}$ (Figure 6 and Table I).

\subsection{Modeling}

The experimental observations presented in the earlier sections were further examined via computational modeling with the following principal objectives: 
1. Past work on Ti-Mo alloys has shown that addition of 9at\%Mo prevented the full collapse of $\{222\}_{\beta}$ planes needed to form an ideal $\omega$ structure $[31,37]$. Therefore, the observation of an ideal $\omega$ structure in water quenched Ti-19at.Vis rather intriguing. This observation will be rationalized using density functional theory (DFT) based calculations.

2. Post annealing solute enrichment $(30-40 \mathrm{at} \% \mathrm{~V})$ within the $\beta$ matrix will strain the $b c c$ lattice, and the resulting $\omega / \beta$ misfit strains will govern the morphological evolution of isothermal $\omega$ precipitates [7]. DFT computations have been employed to investigate the underlying physical basis for such lattice straining, and its consequent influence of the morphological evolution of $\omega$ precipitates has been explained using continuum microelasticity calculations.

\subsubsection{Examination of bcc $(\beta)$ phase lattice distortions using Density Functional Theory}

This section examines the role of $\mathrm{V}$ enrichment in the $\beta$-matrix on the stability of the $\omega$ phase in the WQ condition and under straining of the bcc lattice during latter stages of annealing. We perform DFT calculations on 96 atom supercells with different V contents. Two perspective views the unit cell used in our calculations are schematically shown in Figure 7(a) (shown with two different perspective views), where the atoms marked " $X$ " lie on the $\{222\}_{\beta}$ of the bcc lattice. They also reveal the orientation relationship between the tetragonal unit cell (blue atoms) and the "normal" cubic bcc lattice (green atoms). Atomic positions (blue atoms) of the three atom $\omega$ unit cell are $(0,0,0),(1 / 3,2 / 3,1 / 3+\mathrm{z})$ and $(2 / 3,1 / 3,2 / 3-\mathrm{z})$ where $0<\mathrm{z}<1 / 6$, and the " $\mathrm{X}$ " atoms 
occupy the last two position. Additionally, the unit cell shares a common tetragonal symmetry with the ideal $\omega$ structure, because the " $X$ " atoms lie half way along the length of the unit cell $(\mathrm{z}=1 / 6)$ in $\omega$. A similar tetragonal unit cell was also used in other studies [31, 40, 46, 57]. Finally, DFT calculations were carried out on the basis that increasing $\mathrm{V}$ enrichment will increase the population of $\mathrm{V}-\mathrm{V}$ first nearest neighbors and thereby reduce the $\mathrm{V}-\mathrm{V}$ interatomic distances at higher concentration levels. Consequently, 96 atom supercells with concentration levels 17at.V(Figure 7(b)) and 46at.V(Figure 7(c)) were selected as the initial structures by substituting Ti atoms with V, such that the V-V interatomic distances in the initial 17at.Vand 46at.Vsupercells ranged from $5.4-9.2 \AA$ and 2.8 - 5.6 $\AA$ respectively. These two supercells also correspond to comparable Ti-V compositions reported in the literature [20], where one of them experimentally exhibited $\omega$ formation $(17 \mathrm{at} \% \mathrm{~V})$ upon WQ, while the other did not $(46 a t \% V)$. Subsequently, the ionic, shape and volume degrees of freedom of these supercells were replaced until global energy converged to below $\sim 1 \mathrm{meV} /$ atom and the Hellman-Feynman forces were $<\sim 1 \mathrm{meV} /$ atom..

The energy minimized / relaxed structures for 17at.Vand 46at.Vsupercells are marked as "minimized" in Figure 7(b) and Figure 7(c) respectively. The calculations indicated a fully collapsed $\omega$ in the minimized 17at.Vsupercell (Figure 7(b)), while a rather distorted bcc structure is retained in the energy minimized 46at.Vsupercell (Figure 7(c)). The distorted bcc structure in Figure 7(c) was further confirmed by using common neighbor analyses [58,59]. Crucially, the structure obtained by minimizing 17at.Vsupercell is also consistent with our experimental observations on WQ Ti19at.Valloy which exhibited a fully-collapsed $\{222\}_{\beta}$ plane resulting in ideal $\omega$ 
precipitates (Figure 1) indicating the possibility of a similar $\omega$ structure at lower V compositions.

Electronic structures of the atomic bonds in energy minimized 17at.Vand 46at.Vsupercells were examined in greater detail by calculating the charge density difference $\left(\Delta \rho\right.$ in $\left.\mathrm{e}^{-} / \AA^{3}\right)$ for each supercell. The $\Delta \rho$ was calculated using the expression:

$$
\Delta \rho=\left(\rho_{\mathrm{Ti}-\mathrm{xV}, \mathrm{N}}-\rho_{\mathrm{Ti}, \mathrm{N}}\right)
$$

where $\rho_{\mathrm{Ti}-\mathrm{xV}, \mathrm{N}}$ is the valence charge density of the minimized supercell containing $\mathrm{Ti}$ and $\mathrm{V}$ atoms, and $\rho_{\mathrm{Ti}, 96 \mathrm{~N}}$ is the valence charge density of the same supercell with all the $\mathrm{V}$ atoms replaced by $\mathrm{Ti}$ atoms. Therefore, this methodology permitted us to calculate the excess electron density in the supercell due to the presence of $\mathrm{V}$ atoms. Results of $\Delta \rho$ calculations for 17at.Vand 46at.Vsupercells are shown in Figure7(d) and 7(e) respectively, where the excess electron distribution is indicated with $0.002 \mathrm{e}^{-} / \AA^{3}$ isocharge surface shown in yellow.

In case of the 17at.Vsupercell, the excess electron densities were predominantly localized in discreet pockets around V atoms (Figure 7(d)). A close examination of this supercell (Figure 7(d)) shows the presence of excess charge density between Ti-V atoms as in Ti1- $\mathrm{V}$ and Ti2- $\mathrm{V}$, suggesting a preferential Ti-V bond formation in the $\omega$ structure (marked by straight lines). This conclusion is supported by the minimal presence of excess electron density between V-V atoms in the 17at.Vsupercell. In stark contrast to $17 \mathrm{at} \% \mathrm{~V}$, a different bond description was observed in Ti-46at.Vsystem. Instead of Ti-V bonds, a preferential V-V bond formation (highlighted in Figure 7(e) by red lines) is noticable, because the excess charge density only envelops the vanadium atoms while forming an interconnected network (e.g. V1 and V2 in Figure 10(d)). 
Examination of the relaxed 46at.Vsupercell further revealed that the $\mathrm{V}-\mathrm{V}$ bond lengths have contracted compared to initial unrelaxed configuration, as in Figure 7(e) $\mathrm{d}_{[\mathrm{V} 1-\mathrm{V} 2] \text { Minimized }}=2.595 \AA$, which is a $\sim 8 \%$ reduction from the initial un-relaxed state $\left(\mathrm{d}_{[\mathrm{V} 1-}\right.$ V2]Initial $=2.819 \AA$ ). However, contraction in the bond lengths was not uniform through out the relaxed supercell due to inhomogeneous distortions in the relaxed $b c c$ lattice. By measuring the bond lengths of atoms oriented along $<111>\beta$ (e.g. length of Ti1 - V2 in Figure $10(d)$ and using $d_{[\text {Ti1-V2] }}=a_{\beta} \sqrt{ } 3$ ), the average lattice parameter was estimated as $3.16 \AA$. As a comparison, the reported experimental values of the $\beta$ phase lattice parameter of Ti-19at.Vand Ti-46at.Valloys are 3.23 and $3.16 \AA$ respectively [46]. The computed average $a_{\beta}$ value, and its decrease with increasing $\mathrm{V}$ concentration is in fair agreement with experimental measurements. Our DFT computations indicated that, at higher solute concentration and reduced $\mathrm{V}-\mathrm{V}$ interatomic distance, the preferential formation and contraction of $\mathrm{V}-\mathrm{V}$ bonds causes a reduction in the lattice parameter of the $\beta$ matrix.

Findings from the DFT calculations can be summarized as follows :

1. At low solute concentrations $(17-20 \% \mathrm{~V})$ where the $\mathrm{V}-\mathrm{V}$ atomic separation is large, a preference for $\mathrm{Ti}-\mathrm{V}$ bonds rather than $\mathrm{V}-\mathrm{V}$ bonds results in full collapse of the $\{222\}_{\beta}$ and drives the formation of an ideal $\omega$ structure.

2. At higher solute concentrations $(30-46$ at $\% \mathrm{~V})$ a preference for $\mathrm{V}-\mathrm{V}$ bond accompanied by $\mathrm{V}-\mathrm{V}$ bond contraction is preferred over Ti-V bonding. Comparison of charge distributions in the supercells with lower (17 at\%V) and higher $(46 \mathrm{at} \% \mathrm{~V}) \mathrm{V}$ concentrations showed that the electron density between $\mathrm{V}-\mathrm{V}$ bonds (Figure 7(d)) is greater than Ti-V bonds (Figure 7(b)) indicates a covalent 
character of the bonds [60]. Consequently, the V-V bonds are stiffer compared to the Ti-V bonds [60], which stabilizes the distorted $b c c$ lattice instead of the $\omega$ structure.

3. The contraction of $\mathrm{V}-\mathrm{V}$ bonds, due to enhanced localized charge density, reduces the lattice parameter of the $\beta$ matrix at higher solute concentrations.

\subsubsection{Evolution of $\omega$ upon annealing: Microelasticity results}

In view of the high-resolution experimental observations and DFT calculations reported in previous sections, it is crucial to understand if lattice distortions induced by increasing solute enrichment of the $\beta$ will have an influence on the orientation of the habit plane of

$\omega$ during annealing. The role of $\beta$ lattice contractions on the micromechanics of phase transformation and the attendant crystallography of $\omega$ precipitation during annealing also needed further investigation. For example, earlier studies on Ti-20at.\%V (comparable to our Ti-19at.\%V alloy) by Blackburn and Williams indicated that the faces of cuboidal $\omega$ are parallel to $(001)_{\beta}$ planes $[7,11]$. We investigated these outstanding questions using continuum microelasticity they derived elastic strain energy. We account for the experimentally determined $\beta$ concentrations and $\beta$-matrix as a function of annealing time.

The orientation relationship (OR) between the $\beta$ matrix and $\omega$ precipitate is $\{111\}_{\beta} \|\{0001\}_{\omega}$ and $[1 \overline{1} 0]_{\beta} \|[2 \overline{1} \overline{1} 0]_{\omega}$. Because of symmetry breaking during the $\beta$ $\rightarrow \omega$ transformation, there are four possible orientation variants of $\omega$ phase that can precipitate within the parent $\beta$ phase [7-11]. During the subsequent aging process, the development of isothermal $\omega$ from the athermal $\omega$ is accompanied with the attendant 
partitioning of alloying elements between the two phases, which results in the change of lattice misfit between the two phases owing to the dependence of lattice parameters of on solute content [46], as shown in the Table 1.

According to the lattice correspondence and assuming a fully coherent transformation, the corresponding stress free transformation strain (SFTS) that characterizes the lattice misfit due to $\beta \rightarrow \omega$ transformation can be obtained and expressed with respect to its principle reference axes as

$$
\epsilon_{i j}^{T 0}=\left[\begin{array}{ccc}
\frac{a_{\omega}-\sqrt{2} \cdot a_{\beta}}{\sqrt{2} \cdot a_{\beta}} & 0 & 0 \\
0 & \frac{a_{\omega}-\sqrt{2} \cdot a_{\beta}}{\sqrt{2} \cdot a_{\beta}} & 0 \\
0 & 0 & \frac{2 c_{\omega}-\sqrt{3} \cdot a_{\beta}}{\sqrt{3} \cdot a_{\beta}}
\end{array}\right]
$$

where $a_{\omega}$ and $c_{\omega}$, and $a_{\beta}$ are the equilibrium lattice parameters of the $\omega$ and $\beta$ phases in the Ti-V system. To correspond with the experimental measurements, the principle reference axes of the SFTS for the $\omega$ variant are selected in such a way that the $x$ direction corresponds to $[1 \overline{1} 0]_{\beta} \|[2 \overline{1} \overline{1} 0]_{\omega}$, the $y$ direction corresponds to $[11 \overline{2}]_{\beta} \|$ $[01 \overline{1} 0]_{\omega}$, and $z$ corresponds to $[111]_{\beta} \|[0001]_{\omega}$. The SFTS values, listed in Table II, were calculated using Eq. 2 and with $\mathrm{V}$ concentration dependent $\omega$ and $\beta$ lattice parameters from Ref \#46.

With the information of SFTS, the coherency elastic strain energy density characterized by $B_{p q}(\mathbf{n})$ can be obtained and, in particular, the habit plane orientation, $\mathbf{n}_{0}$, can also be predicted by finding the minimum of $B_{p q}\left(\mathbf{n}_{0}\right)[48,61.] . B_{p q}(\mathbf{n})$ is defined as

$$
B_{p q}(\mathbf{n})=C_{i j k l} \epsilon_{i j}^{T 0}(p) \epsilon_{k l}^{T 0}(q)-n_{i} \sigma_{i j}^{T 0}(p) \Omega_{j k}(\mathbf{n}) \sigma_{k l}^{T 0}(q) n_{l}
$$


where $C_{i j k l}$ is the elastic constant, $\epsilon_{i j}^{T 0}(p)$ is the SFTS for the $p^{t h} \omega$ variant, $\sigma_{i j}^{T 0}(p) \equiv$ $C_{i j k l} \epsilon_{i j}^{T 0}(p), \quad\left[\Omega_{i k}(\mathbf{n})\right]^{-1} \equiv C_{i j k l} n_{j} n_{l}$ is the inverse of the Green's function in the reciprocal space, and $\mathbf{n}$ is a unit vector in the reciprocal space with $n_{i}$ being its $i^{\text {th }}$ component. The influence of $\mathrm{V}$ concentration on the elastic stiffness constants of the $\beta$ matrix have been considered in the calculations. In particular, $\mathrm{C}_{11} \mathrm{C}_{12}$ and $\mathrm{C}_{44}$ (in Voigt notation) were extracted from Ref [62] and their values are listed in Table I. Note that in each case the elastic constants satisfy the well-known Born stability criterion for cubic crystals, i.e. $\mathrm{C}_{44}>0, \mathrm{C}_{11}-\mathrm{C}_{12}>0$ and $\mathrm{C}_{11}+2 \mathrm{C}_{12}>0$ [63]. For a given $\mathrm{V}$ concentration modulus of $\omega$ precipitates were assumed to have the same elastic modulus as that of the matrix, i.e. the homogeneous modulus case [61].

The calculated strain energy density $B_{p q}(\mathbf{n})$ for WQ and annealed conditions are plotted in polar coordinates in Figure 8(a) and 8(b) respectively. Table II shows that lattice parameter of $\omega$ and $\beta$ phases for each heat treatment condition, where the lattice parameters were obtained from the dependence of such values (Aurello et.al. [46]) on the measured composition (Table I). The results suggest clear differences in the habit planes of quenched-in and isothermal $\omega$. In case of quenched-in $\omega$, the strain energy minima $B_{p q}\left(\mathbf{n}_{0}\right)$ occur along [111] $]_{\beta}$ (indicated with arrow in Figure 8(a)), while the strain energy minima for isothermal $\omega$ appears near $[001]_{\beta}$ (arrow in Figure $8(\mathrm{~b})$ ). Furthermore, the $B_{p q}\left(\mathbf{n}_{0}\right)$ values (Table II) for quenched-in $\omega$ is several orders of magnitude lower than that for isothermal $\omega$, due to much lower misfit strain (compare $\epsilon_{1}$ and $\epsilon_{3}$ for WQ and annealed conditions in Table II). During the evolution of $\omega$, the strain energy also increases with annealing time (1, 64 and $256 \mathrm{~h})$, but the increment is not as significant when compared to the WQ condition. Furthermore, our experimental observations 
indicate that the $\omega$ particles in the WQ condition have their long axis oriented along $\langle 111\rangle_{\beta}$ (Figure 1). Microelasticity calculations suggest a well-defined habit plane of $\{111\}_{\beta}$. Considering the small size $(\sim 7 \mathrm{~nm}$ along the long axis, Figure $1(\mathrm{~b}))$ of the $\omega$ particles and also the relatively small misfit strain (as compared to that for $\omega$ particles after isothermal aging) in the water quenched condition, the interfacial energy anisotropy may have played a dominant role in determining the shape of water-quenched $\omega$ particle (Table II).

\section{Discussion}

The present study leads to some new insights into the formation of quenched-in $\omega$ precipitates and their subsequent evolution (compositional, morphological and crystallographic) during isothermal annealing at $300^{\circ} \mathrm{C}$. Furthermore, the coupled experimental and DFT-based computation analysis of the $\beta+\omega$ microstructures at different stages of isothermal annealing allowed us to describe the free energies of the constituent phases ( $\beta$ and $\omega$ ) as a function of an order parameter and solute composition.

The fully collapsed $\{222\}_{\beta}$ planes in quenched-in $\omega$ precipitates obtained from atomic resolution HAADF-STEM (Figure 1) and the bond descriptions revealed by the DFT calculations (Figure 7), together, indicated that a directional Ti-V bonding (rather than $\mathrm{V}-\mathrm{V}$ bonds) is a crucial requirement for the full collapse of $\{222\}_{\beta}$ planes forming the ideal $\omega$ structure. Furthermore, comparison of excess electron charge distribution in the $\beta$ and $\omega$, showed that the $V-V$ bonds (Figures 7(d)) tend to have higher excess charge densities compared to the Ti-V bonds (Figures 7(e)); in other words, the V-V bonds are stronger / stiffer than Ti-V bonds. This key result gives a crucial insight regarding $\omega$ 
formation via the use of a Landau polynomial formalism for order-disorder transformations, which was previously used by Cook to describe the free energy associated with the collapse of $\{222\}_{\beta}$ planes [24-27]. Cook expressed the free energy as $F=A \eta^{2}+B \eta^{3}+C \eta^{4}+\ldots$, where $\eta$ is the order parameter describing the displacement of $\{222\}_{\beta}$ planes and $A, B, C$ are constants related to interplanar coupling [21, 24-27]. Such a schematic version of the F vs. $\eta$ plot for a $\omega$-forming $\beta$-alloy is shown in blue in Figure 9. Taking the first derivative of $F$ with respect to $\eta$, and ignoring higher order terms, we get $\frac{\partial F}{\partial \eta}=A \eta$, where, as a first order harmonic approximation, $A$ becomes the interplanar stiffness (comparable to $f=k \cdot x$, where $f, k$, and $x$ are the force, spring constant/stiffness and displacement respectively [60,64]). Therefore, $A$ will be influenced by the interatomic bond strengths existing within the alloy; meaning stiffer bonds can potentially change the free energy landscape. This is schematically illustrated in Figure 9 using the plot shown in red, where the presence of an excess population of stiff $\mathrm{V}-\mathrm{V}$ bonds will eliminate the free energy minima corresponding to $\omega$, and stabilize the $\beta$ phase. Free energy profiles indicating similar features have been previously revealed by DFT-based computations in Ti-Mo alloys of varying concentrations [31]. Thus, larger population of $\mathrm{V}-\mathrm{V}$ first nearest neighbors and the corresponding $\mathrm{V}-\mathrm{V}$ bond stiffness, will prevent the formation of ideal $\omega$ by restricting the displacement of atoms lying on $\{222\}_{\beta}$. In essence, the DFT results presented in this paper - in conjunction with the experimental data - clearly establish the electronic origins of omega formation.

The coupled TEM and APT examination of $\omega$ precipitates provided new insights regarding the formation of this phase during quenching and subsequent isothermal 
annealing. Three key observations were noted during the isothermal evolution of $\omega$ precipitates. Firstly, early stages of annealing yielded a volume fraction of isothermal $\omega$ precipitates comparable to the volume fraction of $\omega$ precipitates in the as-quenched sample (Figures 3), which suggests that the quenched-in $\omega$ may have an influence on the formation of isothermal $\omega$ precipitates. Secondly, even at the early stages of annealing (10 mins and $1 \mathrm{Hr}$ ), the $\omega$ precipitates exhibited a near-equilibrium solute concentration ( $\sim 5$ at $\%$ referring to Table $\mathrm{I})$; indicating that there is rapid rejection of $\mathrm{V}$ from the $\omega$ precipitates during isothermal annealing. Thirdly, both the quenched-in $\omega$ and the isothermal $\omega$ have the same hexagonal structure (symmetry $\mathrm{P} 6 / \mathrm{mmm}$ ), and differ only in their respective solute content (Table I). These three insights suggest that it is microstructurally difficult to distinguish between quenched-in and "isothermal" $\omega$ in $\beta$-Ti alloys, which form fully collapsed $\omega$ (hexagonal structure) upon water quenching. Furthermore, isothermal annealing of such quenched-in $\omega$ leads to substantial compositional partitioning between $\omega$ and $\beta$ phases, thereby increasing the misfit strains (Table II) due to $\beta$ lattice distortions induced via $\mathrm{V}$ enrichment in the matrix (from DFT calculations, Figure 7(c)). As a result, significantly higher strain energies are involved (refer to Table II) in the case of the $\omega$ precipitates after annealing, resulting in the development of a cuboidal morphology of these precipitates with a $\{001\}_{\beta}$ habit plane (Figure 8(b))

\section{Summary}


The mechanism of $\omega$ precipitation and its growth during isothermal annealing, in a high misfit binary Ti-20wt\%V or Ti-19at.\%Valloy, was systematically investigated by coupling high resolution characterization tools like aberration-corrected HAADF-STEM and atom probe tomography with computations involving ab initio DFT and continuum microelasticity theory. The salient results of our work are:

1. Established the athermal nature of the formation mechanism associated with these quenched-in $\omega$ precipitates. We provided direct experimental evidence showing ideal $\omega$ crystal structure corresponding to fully collapsed $\{222\}_{\beta}$ planes without any compositional partitioning in the water quenched condition.

2. Early stages of annealing $\left(300^{\circ} \mathrm{C} / 10 \mathrm{mins}\right)$ resulted in the formation of ellipsoidal isothermal $\omega$ precipitates. The volume fraction of these isothermal $\omega$ precipitates was comparable to the quenched-in athermal $\omega$ precipitates, and they also exhibited a substantially lower solute content $(\sim 5$ at. $\% \mathrm{~V})$ as compared to the congruent athermal $\omega$. At the latter stages of annealing $\left(300^{\circ} \mathrm{C} / 64\right.$ and $\left.256 \mathrm{H}\right)$, the isothermal $\omega$ precipitates evolved into a cuboidal morphology. Interestingly, the growth of isothermal $\omega$ was associated with solute enrichment in the $\beta$ phase to very high levels of V content (e.g. $\sim 40$ at. $\%$ after $256 \mathrm{H}$ annealing at $300^{\circ} \mathrm{C}$ ). On the other hand, the composition of isothermal $\omega$ observed at the later stages of annealing $(1,64$ and $256 \mathrm{H})$ did not show any discernable change from those formed at after10 mins of annealing, suggesting that these isothermal $\omega$ precipitates achieve near-equilibrium compositions at very early stages.

3. DFT-based ground state structure calculations showed that $\omega$ in the water quenched condition congruently forms $(\omega$ composition $\sim 20$ at. $\% \mathrm{~V})$ via 
preferential Ti-V bonding. Subsequent to long-term isothermal annealing, these $\omega$ precipitates reject $\mathrm{V}$ solute into the $\beta$ matrix, whose composition was experimentally found to be as high as $\sim 50$ at. $\% \mathrm{~V}$. At high solute concentrations ( 50 at.\% $\mathrm{V}$ ) stiffer $\mathrm{V}-\mathrm{V}$ bonds stabilize the $\beta$ phase, and the attendant contraction in the V-V bond lengths not only reduces the lattice parameter, but also significantly distorts the $b c c-\beta$ lattice. Such lattice distortions, produced from isothermal annealing, cause the $\omega$ precipitates to acquire a cuboidal morphology with habit planes aligned parallel to the $\{001\}_{\beta}$ planes.

\section{Acknowledgement}

The authors would like to acknowledge funding from National Science Foundation under DMREF grant DMR-1435611 and also grants DMR-1309270 and DMR-1309277. The authors would also gratefully acknowledge the use of experimental facilities at the Center for Advanced Research and Technology (CART) at the University of North Texas (UNT) and the Center for Electron Microscopy and Analysis (CEMAS). DC and SGS acknowledges computing provided by the high-performance computing facilities at UNT (Talon 2) and Texas Advanced Computing Center (TACC), while RS and YW acknowledges the support from the computing cluster at the Ohio Supercomputing Center. RS and YW also would like to thank financial support from the National Science Foundation under the DMREF program with Grant No. DMR-1435483. 


\section{References}

1. Usikov, M. P., \& Zilbershtein, V. A. (1973). The orientation relationship between the $\alpha$ - and $\omega$ - phases of titanium and zirconium. Physica status solidi (a), 19(1), 53-58.

2. Rabinkin, A., Talianker, M., \& Botstein, O. (1981). Crystallography and a model of the $\alpha \rightarrow \omega$ phase transformation in zirconium. Acta Metallurgica,29(4), 691-698.

3. Song, S. G., \& Gray III, G. T. (1995). Microscopic and crystallographic aspects of retained omega phase in shock-loaded zirconium and its formation mechanism. Philosophical Magazine A, 71(2), 275-290.

4. Greeff, C. W., Trinkle, D. R., \& Albers, R. C. (2001). Shock-induced $\alpha-\omega$ transition in titanium. Journal of Applied Physics, 90(5), 2221-2226.

5. Hennig, R. G., Trinkle, D. R., Bouchet, J., Srinivasan, S. G., Albers, R. C., \& Wilkins, J. W. (2005). Impurities block the $\alpha$ to $\omega$ martensitic transformation in titanium. Nature materials, 4(2), 129-133.

6. Banerjee, S., \& Mukhopadhyay, P. (2007). Phase transformations on examples from titanium and zirconium. Pergamon Materials Series, 12, 1-813.

7. Williams, J.C. \& Blackburn, M.J., (1969) Influence of misfit on morphology and stability of omega phase in titanium-transition metal alloys, Trans. Metall. Soc. AIME $2452352-2355$.

8. Rhodes, C. G., \& Williams, J. C. (1975). The precipitation of $\alpha$-phase in metastable $\beta$-phase Ti alloys. Metallurgical Transactions A, 6(11), 2103-2114.

9. Ohmori, Y., Ogo, T., Nakai, K., \& Kobayashi, S. (2001). Effects of $\omega$-phase precipitation on $\beta \rightarrow \alpha, \alpha^{\prime \prime}$ transformations in a metastable $\beta$ titanium alloy.Materials Science and Engineering: A, 312(1), 182-188.

10. Prima, F., Vermaut, P., Texier, G., Ansel, D., \& Gloriant, T. (2006). Evidence of $\alpha-$ nanophase heterogeneous nucleation from $\omega$ particles in a $\beta$-metastable Ti-based alloy by high-resolution electron microscopy. Scripta materialia,54(4), 645-648.

11. Nag, S., Banerjee, R., Srinivasan, R., Hwang, J. Y., Harper, M., \& Fraser, H. L. (2009). $\omega$-Assisted nucleation and growth of $\alpha$ precipitates in the $\mathrm{Ti}-5 \mathrm{Al}-5 \mathrm{Mo}-5 \mathrm{~V}-$ 3Cr-0.5 Fe $\beta$ titanium alloy. Acta Materialia, 57(7), 2136-2147. 
12. Li, T., Kent, D., Sha, G., Dargusch, M. S., \& Cairney, J. M. (2015). The mechanism of $\omega$-assisted $\alpha$ phase formation in near $\beta$-Ti alloys. Scripta Materialia, 104, 75-78.

13. Zheng, Y., Choudhuri, D., Alam, T., Williams, R. E., Banerjee, R., \& Fraser, H. L. (2016). The role of cuboidal $\omega$ precipitates on $\alpha$ precipitation in a Ti-20V alloy. Scripta Materialia, 123, 81-85.

14. Zheng, Y., Williams, R. E., Wang, D., Shi, R., Nag, S., Kami, P., ... \& Fraser, H. L. (2016). Role of $\omega$ phase in the formation of extremely refined intragranular $\alpha$ precipitates in metastable $\beta$-titanium alloys. Acta Materialia,103, 850-858.

15. Devaraj, Arun, Vineet V. Joshi, Ankit Srivastava, Sandeep Manandhar, Vladimir Moxson, Volodymyr A. Duz, and Curt Lavender. "A low-cost hierarchical nanostructured beta-titanium alloy with high strength." Nature communications 7 (2016).

16. Lütjering, G., \& Williams, J. C. (2003). Titanium (Vol. 2). Berlin: Springer.pp 332336

17. Mantri, S. A., Choudhuri, D., Behera, A., Cotton, J. D., Kumar, N., \& Banerjee, R. (2015). Influence of fine-scale alpha precipitation on the mechanical properties of the beta titanium alloy beta-21S. Metallurgical and Materials Transactions A, 46(7), 2803-2808.

18. Hickman, B. S. (1969). The formation of omega phase in titanium and zirconium alloys: a review. Journal of Materials Science, 4(6), 554-563.

19. De Fontaine, D. (1970). Mechanical instabilities in the bcc lattice and the beta to omega phase transformation. Acta metallurgica, 18(2), 275-279.

20. McCabe, K. K., \& Sass, S. L. (1971). The initial stages of the omega phase transformation in Ti-V alloys. Philosophical Magazine, 23(184), 957-970.

21. J. Williams, J. C., Hickman, B. S., \& Leslie, D. H. (1971). The effect of ternary additions on the decompositon of metastable beta-phase titanium alloys.Metallurgical Transactions, 2(2), 477-484.

22. Balcerzak, A. T., \& Sass, S. L. (1972). The formation of the $\omega$ phase in Ti-Nb alloys. Metallurgical Transactions, 3(6), 1601-1605.

23. Williams, J. C., De Fontaine, D., \& Paton, N. E. (1973). The $\omega$-phase as an example of an unusual shear transformation. Metallurgical Transactions,4(12), 2701-2708. 
24. Cook, H. E. (1974). A theory of the omega transformation. Acta Metallurgica,22(2), 239-247.

25. Cook, H. E. (1975). On first-order structural phase transitions-I. General considerations of pre-transition and nucleation phenomena. Acta Metallurgica, 23(9), 1027-1039.

26. Cook, H. E. (1975). On first-order structural phase transitions-II. The omega transformation in Zr-Nb alloys. Acta Metallurgica, 23(9), 1041-1054.

27. Duerig, T. W., Terlinde, G. T., \& Williams, J. C. (1980). Phase transformations and tensile properties of Ti-10V-2Fe-3AI. Metallurgical Transactions A,11(12), 19871998.

28. Duerig, T.W., Terlinde, G.T. \& Williams, J.C. Proc $4^{\text {th }}$ Interl Conf. on Titanium, edited by H Kimura and O Izumi 2 (1980) 1299

29. Moffat, D. L., \& Larbalestier, D. C. (1988). The competition between the alpha and omega phases in aged Ti-Nb alloys. Metallurgical Transactions A, 19(7), 1687-1694.

30. Petry, W., Heiming, A., Trampenau, J., Alba, M., \& Vogl, G. (1989). Strong phonon softening in the BCC phase of titanium. Physica B: Condensed Matter, 156, 56-58.

31. Nag, S., Devaraj, A., Srinivasan, R., Williams, R. E. A., Gupta, N., Viswanathan, G. B., Tiley, J.S, Banerjee, S, Srinivasan, S.G., Fraser, H.L. \& Banerjee, R. (2011). Novel mixed-mode phase transition involving a composition-dependent displacive component. Physical review letters, 106(24), 245701.

32. Sukedai, E., Hashimoto, H., \& Hida, M. (1987). Fine structure of as-quenched omega-phase of Ti-Mo alloy observed by HREM with image processing.Japanese journal of applied physics, 26(6A), L961.

33. Sukedai, E., Liu, W., Awaji, M., \& Horiuchi, T. (1994). Investigation of atomic structure of $\omega$-phase crystals in Ti-Mo alloys using high-resolution electron microscopy. Ultramicroscopy, 54(2), 192-200.

34. Sukedai, E., Kitano, Y., \& Ohnishi, A. (1997). Investigation of initial structures of aged $\omega$-phase crystals in $\beta$-titanium alloys using high resolution electron microscopy. Micron, 28(4), 269-277. 
35. Nellist, P. D., Chisholm, M. F., Dellby, N., Krivanek, O. L., Murfitt, M. F., Szilagyi, Z. S., ... \& Pennycook, S. J. (2004). Direct sub-angstrom imaging of a crystal lattice. Science, 305(5691), 1741-1741.

36. Pennycook, S. J., \& Nellist, P. D. (1999). Z-contrast scanning transmission electron microscopy. In Impact of electron and scanning probe microscopy on materials research (pp. 161-207). Springer Netherlands.

37. Devaraj, A., Nag, S., Srinivasan, R., Williams, R. E. A., Banerjee, S., Banerjee, R., \& Fraser, H. L. (2012). Experimental evidence of concurrent compositional and structural instabilities leading to $\omega$ precipitation in titanium-molybdenum alloys. Acta Materialia, 60(2), 596-609.

38. Devaraj, A., Williams, R. E. A., Nag, S., Srinivasan, R., Fraser, H. L., \& Banerjee, R. (2009). Three-dimensional morphology and composition of omega precipitates in a binary titanium-molybdenum alloy. Scripta Materialia,61(7), 701-704.

39. Hanlumyuang, Y., Sankaran, R. P., Sherburne, M. P., Morris Jr, J. W., \& Chrzan, D. C. (2012). Phonons and phase stability in Ti-V approximants to gum metal. Physical Review B, 85(14), 144108.

40. Tang, B., Cui, Y. W., Chang, H., Kou, H., Li, J., \& Zhou, L. (2012). A phase-field approach to athermal $\beta \rightarrow \omega$ transformation. Computational Materials Science, 53(1), 187-193.

41. Dutta, J., Ananthakrishna, G., \& Banerjee, S. (2012). On the athermal nature of the $\beta$ to $\omega$ transformation. Acta Materialia, 60(2), 556-564.

42. Ghosh, P. S., Arya, A., Tewari, R., \& Dey, G. K. (2014). Alpha to omega martensitic phase transformation pathways in pure Zr. Journal of Alloys and Compounds, 586, 693-698.

43. Yano, T., Uchida, T., Shimizu, Y., \& Takesue, N. (2008). Valence-electronic effect on low-temperature phase stability of the omega phase. Journal of Physics: Condensed Matter, 20(28), 285216.

44. Koul, M. K., \& Breedis, J. F. (1970). Omega phase embrittlement in aged TiV. Metallurgical and Materials Transactions B, 1(5), 1451-1452. 
45. Ng, H. P., Devaraj, A., Nag, S., Bettles, C. J., Gibson, M., Fraser, H. L., ... \& Banerjee, R. (2011). Phase separation and formation of omega phase in the beta matrix of a Ti-V-Cu alloy. Acta Materialia, 59(8), 2981-2991.

46. Aurelio, G., Guillermet, A. F., Cuello, G. J., \& Campo, J. (2002). Metastable phases in the Ti-V system: Part I. Neutron diffraction study and assessment of structural properties. Metallurgical and Materials Transactions A, 33(5), 1307-1317, and the reference therein

47. B. Gault, M.P. Moody, J.M. Cairney and S.P. Ringer, Atom probe microscopy, $1^{\text {st }}$ ed, Springer, New York (2012)

48. A.G. Khachaturyan, Theory of Structural Transformations in Solids, Dover Publications, New York (2008)

49. Choudhuri, D., Dendge, N., Nag, S., Meher, S., Alam, T., Gibson, M. A., \& Banerjee, R. (2014). Homogeneous and heterogeneous precipitation mechanisms in a binary Mg-Nd alloy. Journal of Materials Science, 49(20), 6986-7003.

50. Perdew, J. P., Burke, K., \& Ernzerhof, M. (1996). Generalized gradient approximation made simple. Physical review letters, 77(18), 3865.

51. Kresse, G., \& Hafner, J. (1993). Ab initio molecular dynamics for liquid metals. Physical Review B, 47(1), 558.

52. Kresse, G., \& Hafner, J. (1994). Ab initio molecular-dynamics simulation of the liquid-metal-amorphous-semiconductor transition in germanium. Physical Review $B, 49(20), 14251$.

53. Henkelman, G., Uberuaga, B. P., \& Jónsson, H. (2000). A climbing image nudged elastic band method for finding saddle points and minimum energy paths. The Journal of chemical physics, 113(22), 9901-9904.

54. Hellman, O. C., Vandenbroucke, J. A., Rüsing, J., Isheim, D., \& Seidman, D. N. (2000). Analysis of three-dimensional atom-probe data by the proximity histogram. Microscopy and Microanalysis, 6(05), 437-444.

55. W.F. Gale and T.C. Totmeier, Smithells Metals Reference Book, $8^{\text {th }}$ ed., Elsevier Massachusetts (2004) 
56. Borkar, T., Nag, S., Ren, Y., Tiley, J., \& Banerjee, R. (2014). Reactive spark plasma sintering (SPS) of nitride reinforced titanium alloy composites.Journal of Alloys and Compounds, 617, 933-945.

57. Lai, M. J., Tasan, C. C., Zhang, J., Grabowski, B., Huang, L. F., \& Raabe, D. (2015). Origin of shear induced $\beta$ to $\omega$ transition in Ti-Nb-based alloys. Acta Materialia, 92, $55-63$.

58. Honeycutt, J. D., \& Andersen, H. C. (1987). Molecular dynamics study of melting and freezing of small Lennard-Jones clusters. Journal of Physical Chemistry, 91(19), 4950-4963.

59. Stukowski, A. (2009). Visualization and analysis of atomistic simulation data with OVITO-the Open Visualization Tool. Modelling and Simulation in Materials Science and Engineering, 18(1), 015012.

60. Sutton, A. P. Electronic structure of materials. Clarendon Press, 1993.

61. Shi, R., Ma, N., \& Wang, Y. (2012). Predicting equilibrium shape of precipitates as function of coherency state. Acta Materialia, 60(10), 4172-4184.

62. Li, T., Morris Jr, J. W., Nagasako, N., Kuramoto, S., \& Chrzan, D. C. (2007). "Ideal" engineering alloys. Physical review letters, 98(10), 105503.

63. Born, Max. "On the stability of crystal lattices. I." In Mathematical Proceedings of the Cambridge Philosophical Society, vol. 36, no. 02, pp. 160-172. Cambridge University Press, 1940.

64. Ashcroft, N. W., \& Mermin, N. D. (1976). Introduction to Solid State Physics.Saunders, Philadelphia. 


\section{Figure Captions}

Figure 1. (a) Selected area diffraction pattern (SADP) recorded along [011] $\beta$ of a water quenched specimen and (b) a $\omega$ precipitate delineated in a atomic resolution HAAADFSTEM image viewed along [011]ß. Enlarged views showing (c) showing fully collapsed $\{222\}_{\beta}$ planes in $\omega$, and $(d)$ reference $\beta$ lattice.

Figure 2. Atom probe results from the WQ condition showing: (a) APT tip depicted with $\mathrm{V}$ ion map in red, (b) binomial distribution comparing the observed data points and the distribution expected from a solid solution, and (c) a cylinder position along the tip axis to measure composition profile, and (d) results from compositional profiling.

Figure 3. Dark field (DF) TEM images comparing the size, morphology and distribution of $\omega$ precipitates in (a) WQ condition and subsequent annealing at $300^{\circ} \mathrm{C}$ for (b) $1 \mathrm{~min}$, (c) $10 \mathrm{~min}$ (d) $1 \mathrm{Hr}$, (e) $64 \mathrm{H}$ and (e) $256 \mathrm{H}$. All images are shown in the same magnification. The insets show the corresponding $[011] \beta$ SADPs and the $\beta / \omega$ orientation relationship via their respective zone axis motifs. The circle in the insets indicate the $\omega$ reflection used for recoding the DF images.

Figure 4. Atom probe results from the a $10 \mathrm{~min} / 300^{\circ} \mathrm{C}$ annealed specimen : (a) isothermal $\omega$ precipitates indicated with a isoconcentration surface (or isosurface) with V ion map as background, (b) corresponding solute partitioning across $\beta$ and $\omega$ via proximity histogram, (c) 2D color contour map from a selected region (inset) in the 3D 
reconstruction showing solute depleted pockets of $\omega$, and (d) 1D concentration profile indicating very high solute concentration in $\beta$ regions adjoining the $\omega$ precipitates. (e) Atom probe results, after $1 \mathrm{H} / 300^{\circ} \mathrm{C}$ annealing, showing $\mathrm{V}$ portioning across $\beta / \omega$ interface. In "e" the selected $\omega$ precipitates are presented as inset.

Figure 5. Atom probe results from the a $64 \mathrm{Hr} / 300^{\circ} \mathrm{C}$ annealed specimen : (a) High volume fraction of ellipsoidal shaped isothermal $\omega$ precipitates delineated with isosurfaces, (b) corresponding solute partitioning across $\beta$ and $\omega$ via proxigram, (c) 2D color contour map from selected region (inset) showing solute depleted pockets of $\omega$, and (d) $1 \mathrm{D}$ concentration profile indicating very high solute concentration in $\beta$ regions adjoining the $\omega$ precipitates.

Figure 6. Atom probe results from the a $256 \mathrm{Hr} / 300^{\circ} \mathrm{C}$ annealed specimen : (a) High volume fraction of cuboidal isothermal $\omega$ precipitates delineated with isosurfaces, (b) corresponding solute partitioning across $\beta$ and $\omega$ via proxigram, (c) 2D color contour map from selected region (inset) showing solute depleted pockets of $\omega$, and (d) 1D concentration profile indicating very high solute concentration in $\beta$ regions adjoining the $\omega$ precipitates.

Figure 7. (a) Different perspective views indicating the orientation relation between $\omega$ and the cubic bcc unit cells. The initial and energy minimized 96 atoms supercells containing (b) 17at.Vand (c) 46 at.\%V concentrations. Note that the bcc unit cell relaxed into $\omega$ in case of $17 \mathrm{at} \% \mathrm{~V}$, whereas the $b c c$ unit cell becomes heavily distorted after 
relaxing the 46 at. $\% \mathrm{~V}$ supercell. Electron charge density difference plots indicating excess electron densities, within supercells showing (d) $\omega(17$ at.\%V) and (e) $\beta$ (46 at.\%V) structures. The difference density distribution is indicated in yellow with $0.002 \mathrm{e} /$ $\AA^{3}$ iso-charge surfaces. Ti and $\mathrm{V}$ atoms are indicated with violet and red colors.

Figure 8. Polar plots showing the strain energy densities $B_{p q}(\mathbf{n})$ in (a) water quenched condition and (b) annealing for 1,64 and $256 \mathrm{H}$ at $300^{\circ} \mathrm{C}$.

Figure 9. Schematic showing the change in the curvature of free energy $(\Delta F)$ vs. order parameter $(\eta)$ plots due to increasing tendency for $\mathrm{V}-\mathrm{V}$ bond formation. At lower $\mathrm{V}$ concentrations (e.g. water quenched $\mathrm{Ti}-20 \mathrm{wt} \% \mathrm{~V}$ or $\mathrm{Ti}-19 \mathrm{at} \% \mathrm{~V}$ ), or smaller population of $\mathrm{V}-\mathrm{V}$ bonds, a minima corresponding to quenched-in $\omega$ formation via $\{222\} \beta$ collapse is indicated. In comparison, such minima disappears for higher population of V-V bonds e.g. V-enriched $\beta$ matrix in annealed Ti-20wt. $\% \mathrm{~V}$ or Ti-19at.\%V, or upon water quenching a higher concentration Ti- $\mathrm{V}$ alloy. 


\section{List of Tables}

Table I. List of experimentally determined $\omega$ size, morphology and concentrations of $\omega$ and $\beta$

\begin{tabular}{|c|c|c|c|c|c|c|c|}
\hline \multirow{2}{*}{ Time } & \multirow{2}{*}{$\begin{array}{l}\text { Observed } \omega \\
\text { morphology }\end{array}$} & \multicolumn{2}{|c|}{$\begin{array}{c}\text { Average } \mathrm{V} \text { concentration } \\
\text { (at } \%)\end{array}$} & \multirow{2}{*}{$\begin{array}{c}\text { Peak V } \\
\text { concentration in } \\
\beta \text { regions near } \omega \\
\text { (at } \%)\end{array}$} & \multicolumn{3}{|c|}{$\begin{array}{l}\text { Elastic Constant } \\
\qquad(\mathrm{GPa})^{\mathrm{b}}\end{array}$} \\
\hline & & $\omega$ & $\beta$ & & $C_{11}$ & $C_{12}$ & $C_{44}$ \\
\hline 0 (WQ) & Ellipsoidal & \multicolumn{2}{|c|}{$21.9 \pm 5.0$} & 21.9 & 9.9 & -0.8 & 46.2 \\
\hline 10 mins & Ellipsoidal & $5.4^{\mathrm{a}}$ & $23.4^{\mathrm{a}}$ & 25.0 & 12.2 & -1.0 & 45.7 \\
\hline $1 \mathrm{H}$ & $\begin{array}{l}\text { Ellipsoidal / } \\
\text { spheroidal }\end{array}$ & $5.8^{\mathrm{a}}$ & $22.7^{\mathrm{a}}$ & 30.0 & 17.6 & -1.5 & 44.4 \\
\hline $64 \mathrm{H}$ & $\begin{array}{l}\text { Ellipsoidal - } \\
\text { near cuboidal }\end{array}$ & $5.3^{\mathrm{a}}$ & $33.0^{\mathrm{a}}$ & 40.0 & 28.7 & -2.4 & 42.4 \\
\hline $256 \mathrm{H}$ & Cuboidal & $4.9^{\mathrm{a}}$ & $35.0^{\mathrm{a}}$ & 50.0 & 40.6 & -3.4 & 40.3 \\
\hline
\end{tabular}

${ }^{\mathrm{a}}$ Standard deviation \pm 1.0

${ }^{\mathrm{b}}$ Elastic constants were extracted from Ref\#62

Table II. Lattice parameters of the $\beta$ and $\omega$ phases, stress free transformation strain (SFTS), minimum strain energy density and habit plane orientation as a function of annealing time

\begin{tabular}{|c|c|c|c|c|c|c|c|}
\hline \multirow{2}{*}{ Time (Hrs) } & \multicolumn{3}{|c|}{ Lattice Parameters $(\AA)^{*}$} & \multicolumn{2}{|c|}{ SFTS $\left(\times 10^{-2}\right)^{* *}$} & \multirow{2}{*}{$\begin{array}{c}B_{p q}\left(\mathbf{n}_{0}\right) \\
\left(\times 10^{7} \mathbf{J} / \mathrm{m}^{3}\right)\end{array}$} & \multirow{2}{*}{$\begin{array}{l}\text { Habit Plane Orientation } \\
\qquad \mathbf{n}_{0}\end{array}$} \\
\hline & $a_{\beta}$ & $a_{\omega}$ & $c_{\omega}$ & $\epsilon_{1}\left(=\epsilon_{2}\right)$ & $\epsilon_{3}$ & & \\
\hline $0(\mathrm{WQ})$ & 3.226 & 4.562 & 2.794 & $3.228 \times 10^{-3}$ & $1.601 \times 10^{-2}$ & $2.463 \times 10^{-6}$ & {$[1 ; 1 ; 1]_{\beta}$} \\
\hline 1 & 3.224 & 4.620 & 2.829 & 1.339 & 1.333 & 0.566 & {$[0 ; 0 ; 1]_{\beta}$} \\
\hline 64 & 3.197 & 4.622 & 2.830 & 2.231 & 2.217 & 2.567 & {$[0 ; 0 ; 1]_{\beta}$} \\
\hline 256 & 3.192 & 4.624 & 2.831 & 2.431 & 2.409 & 4.304 & {$[0 ; 0 ; 1]_{\beta}$} \\
\hline
\end{tabular}

Specific indices are assigned in accordance with the particular variant of OR $(111)_{\beta} \|(0001)_{\omega}$ and $[1 \overline{1} 0]_{\beta} \|[2 \overline{1} \overline{1} 0]_{\omega}$ 

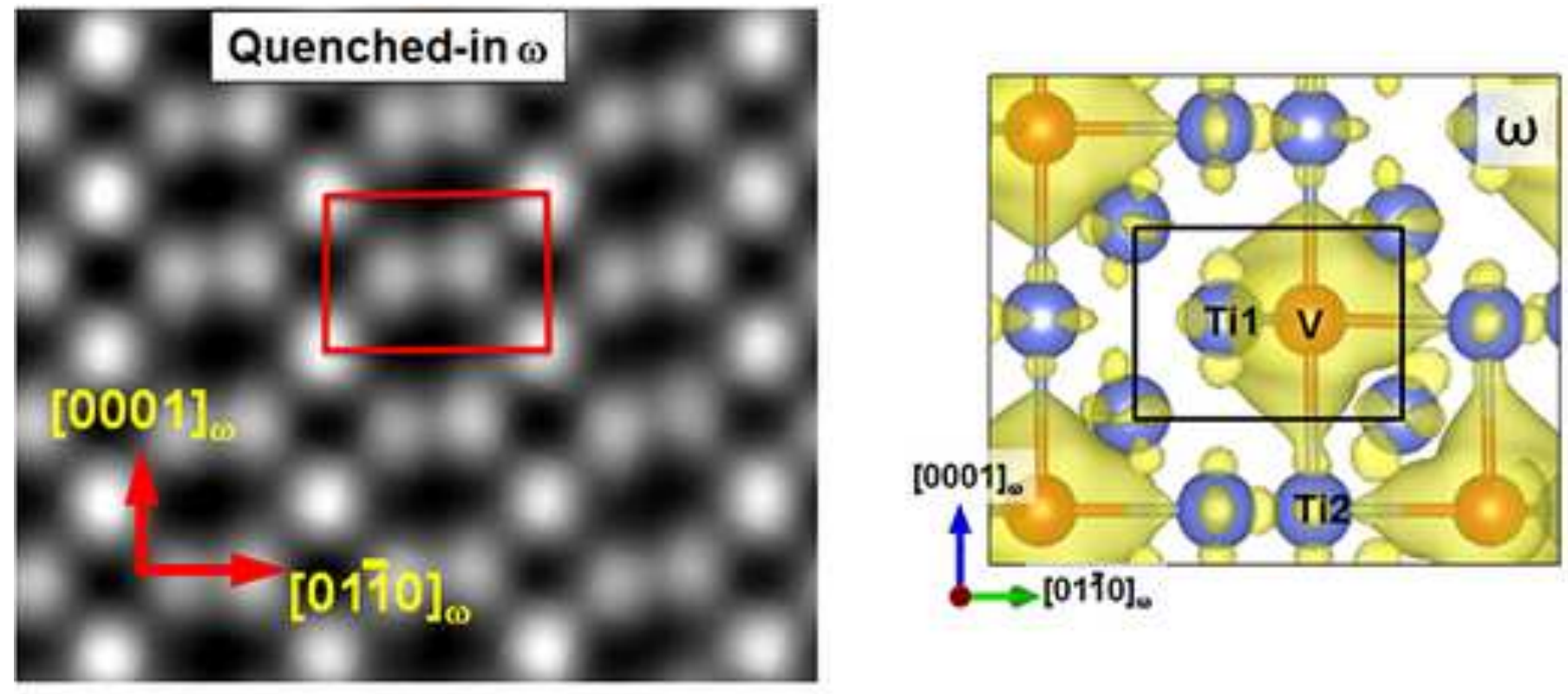

Isothermal annealing

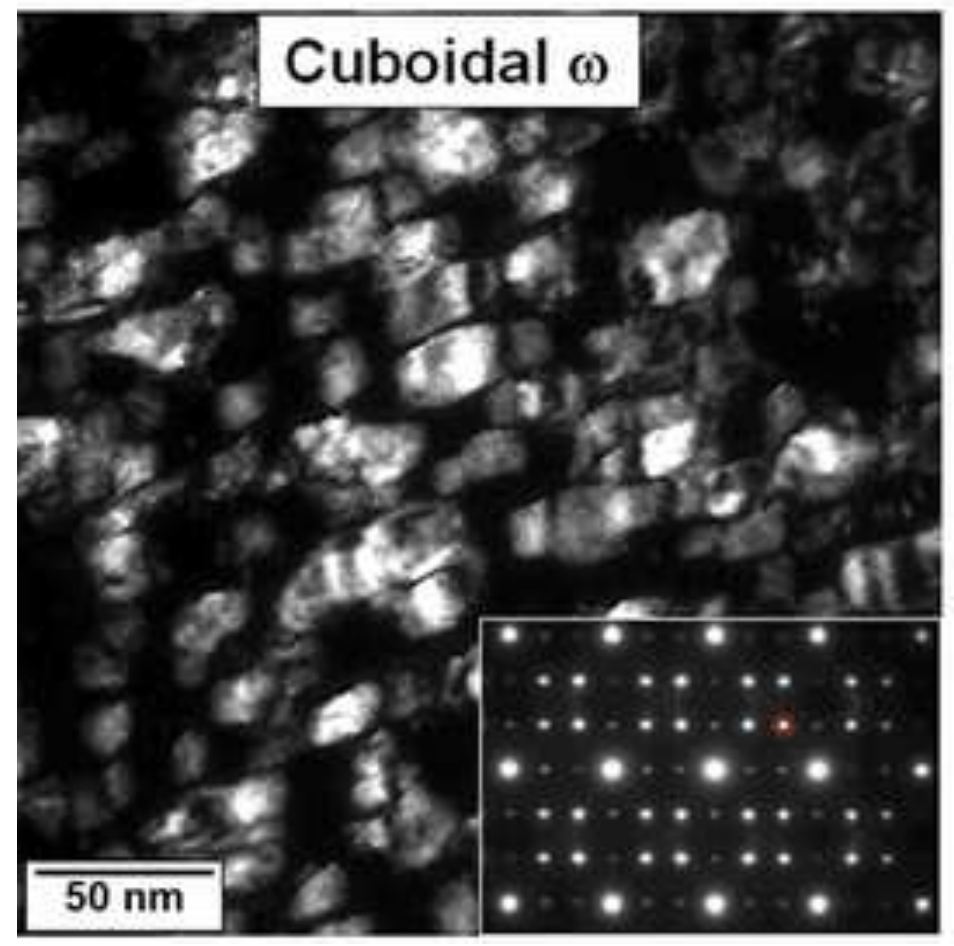

(b) $B_{p 0}(\mathbf{n})$ on $(1 \overline{10})_{\beta}$ Plane $\left(\times 10^{7} . J / m^{3}\right)$

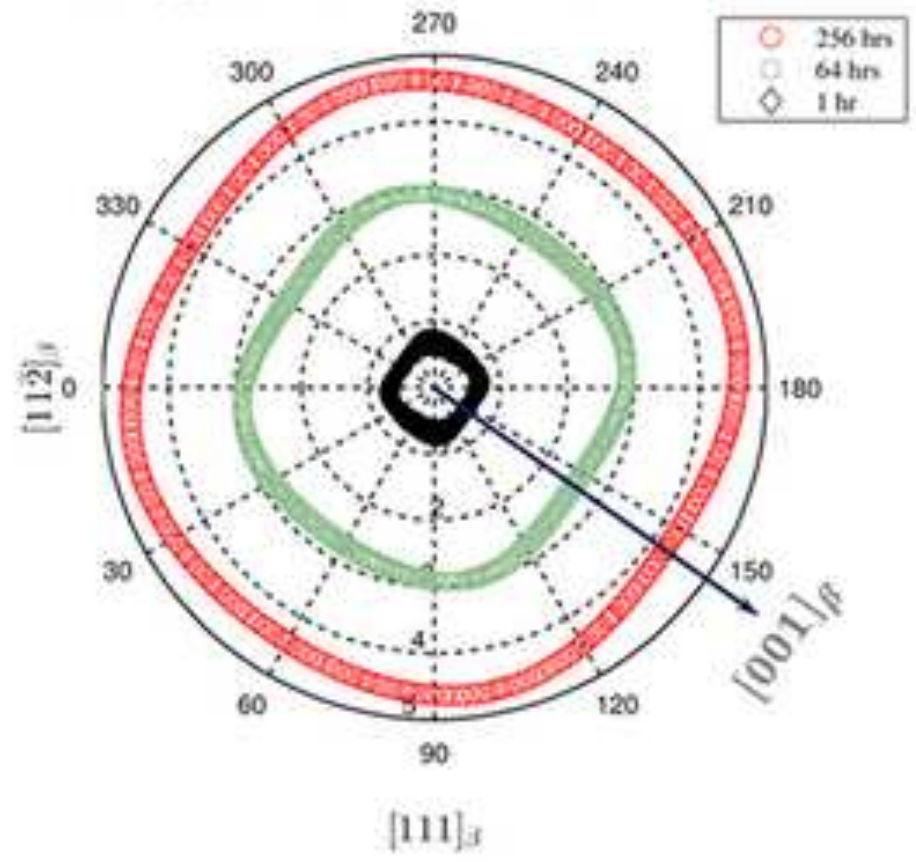




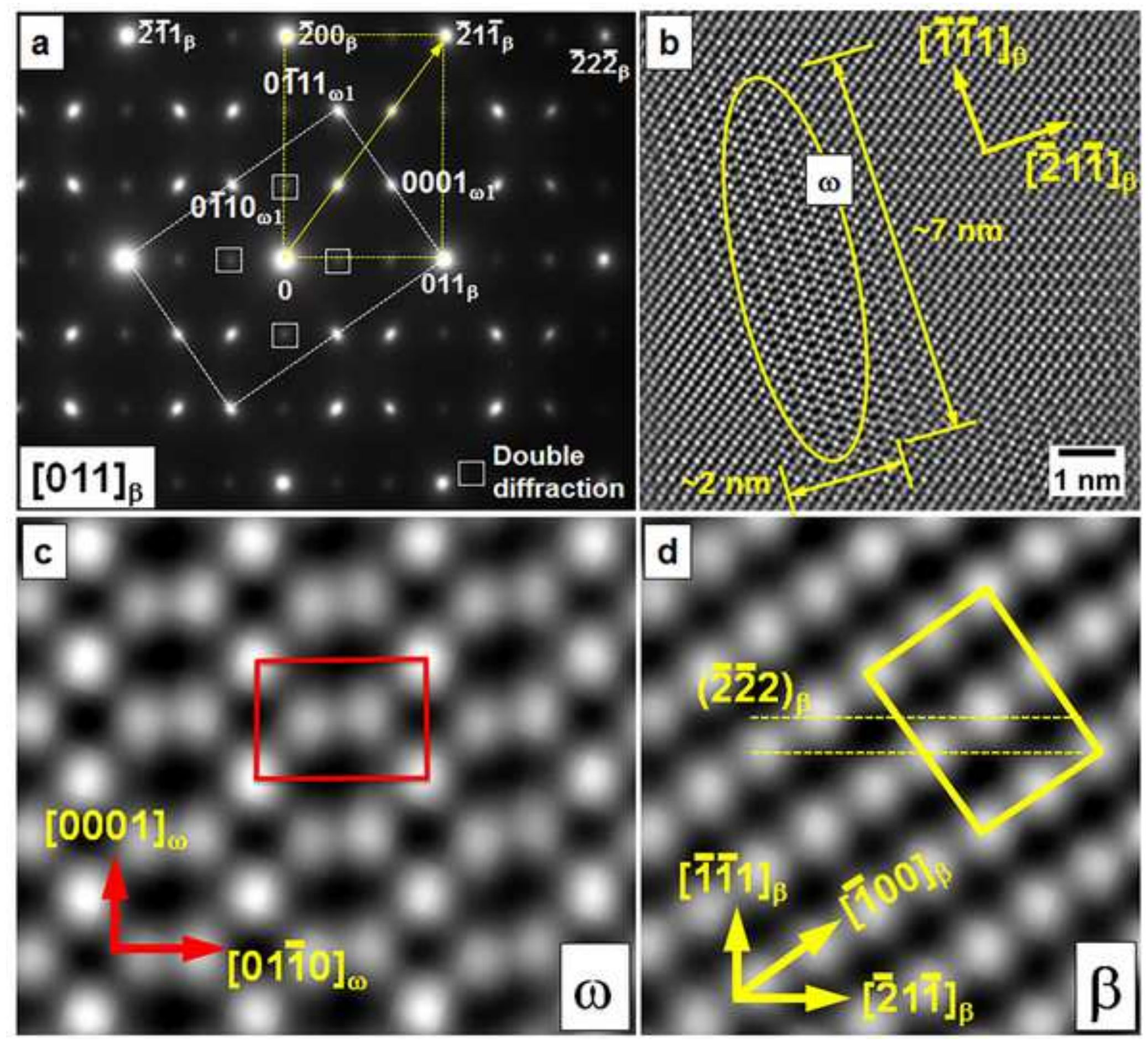



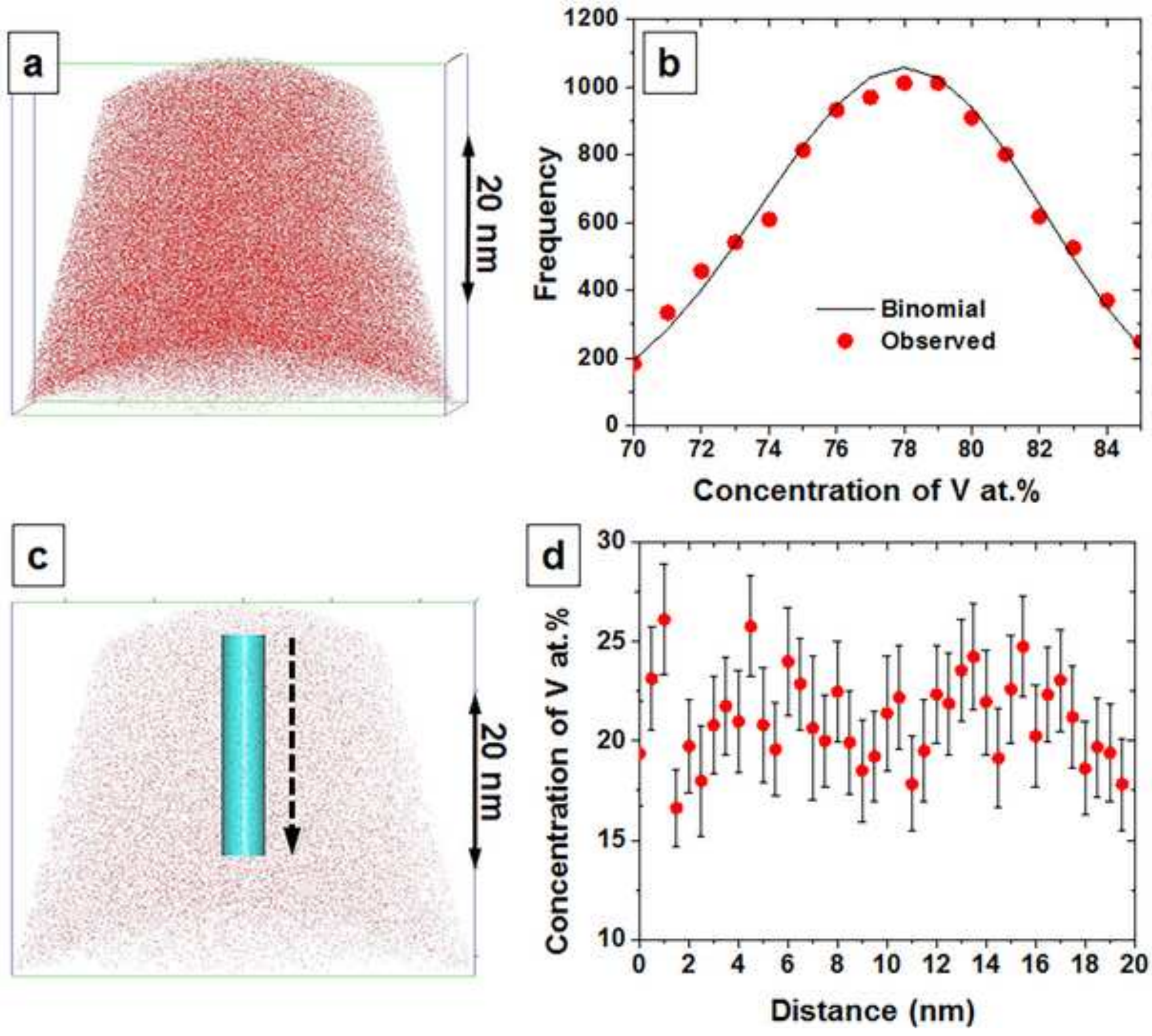

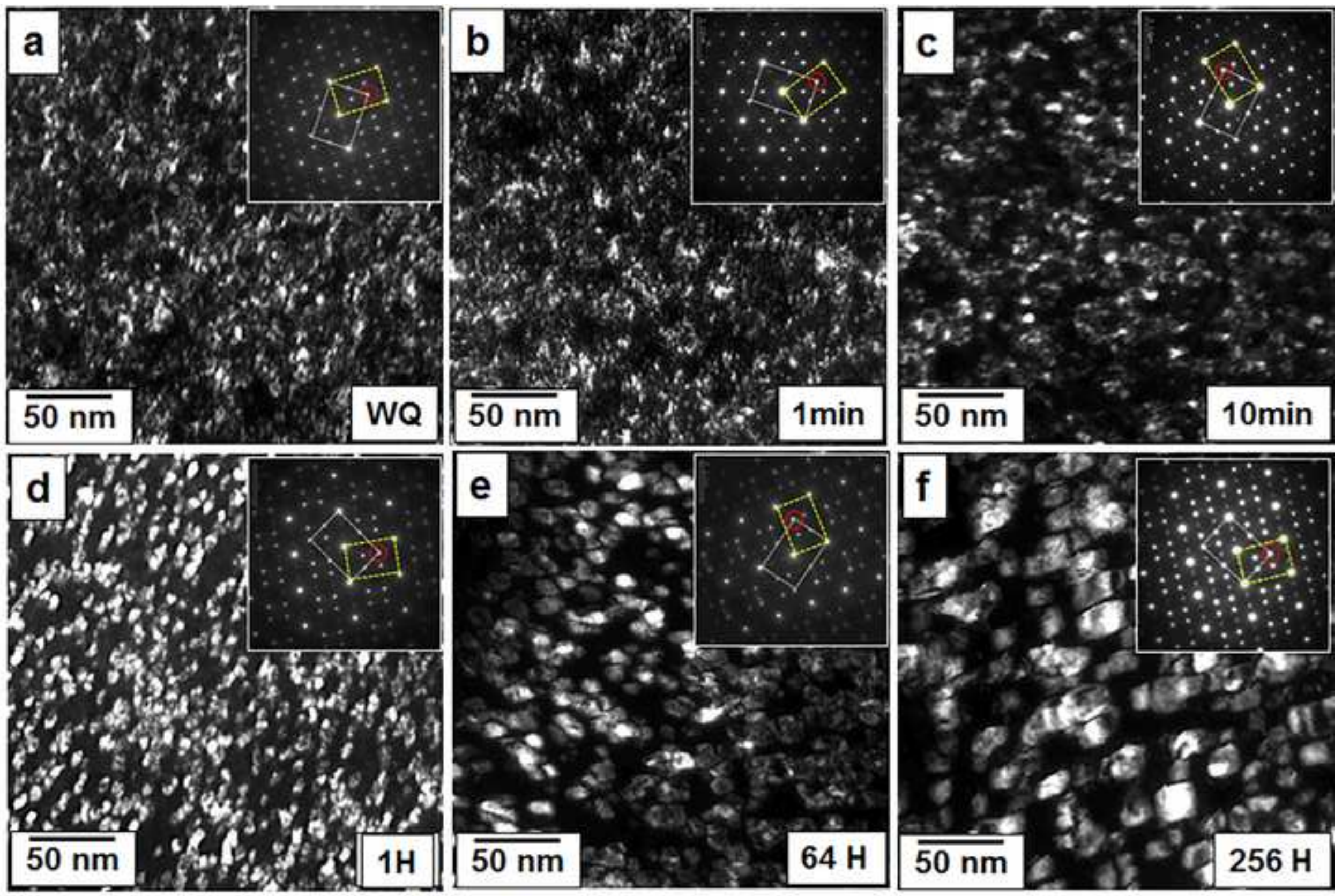

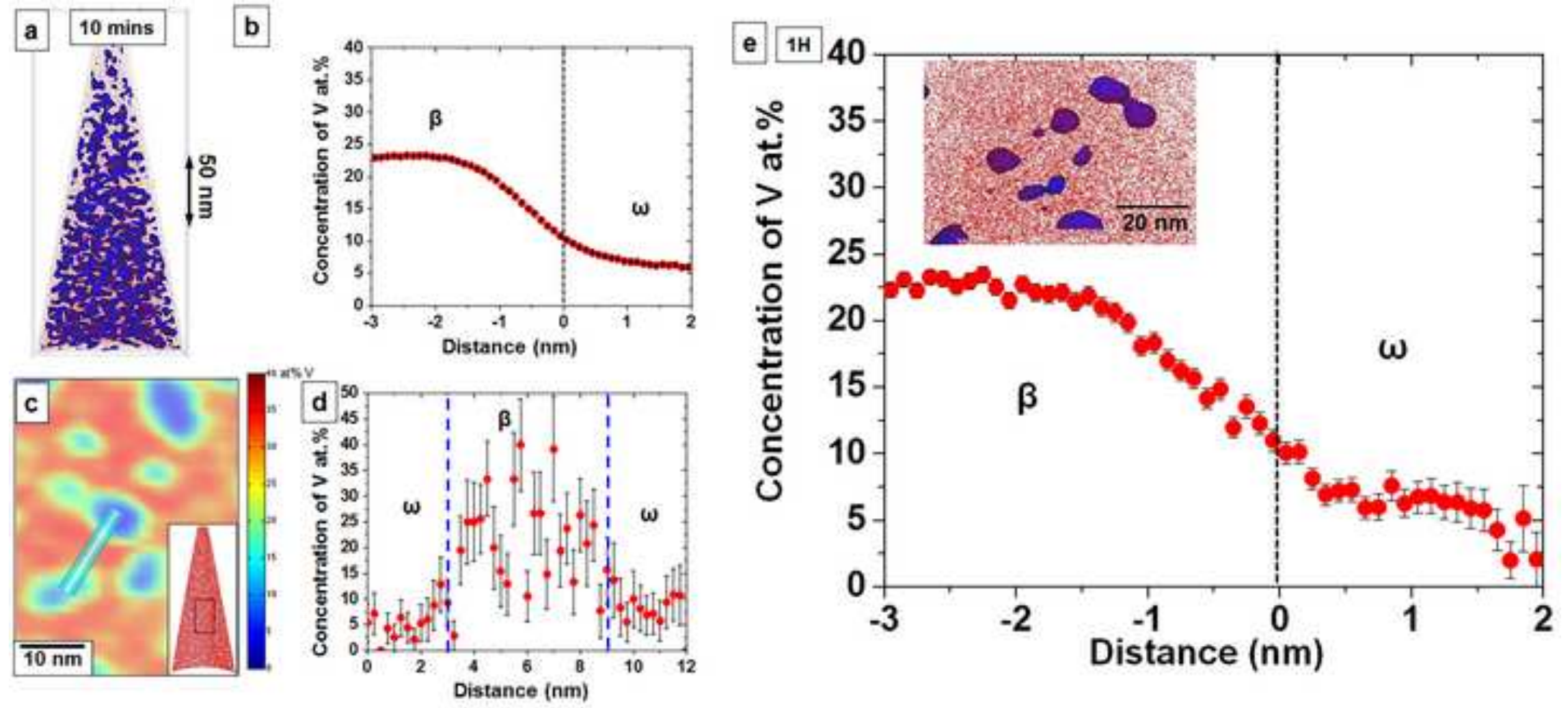

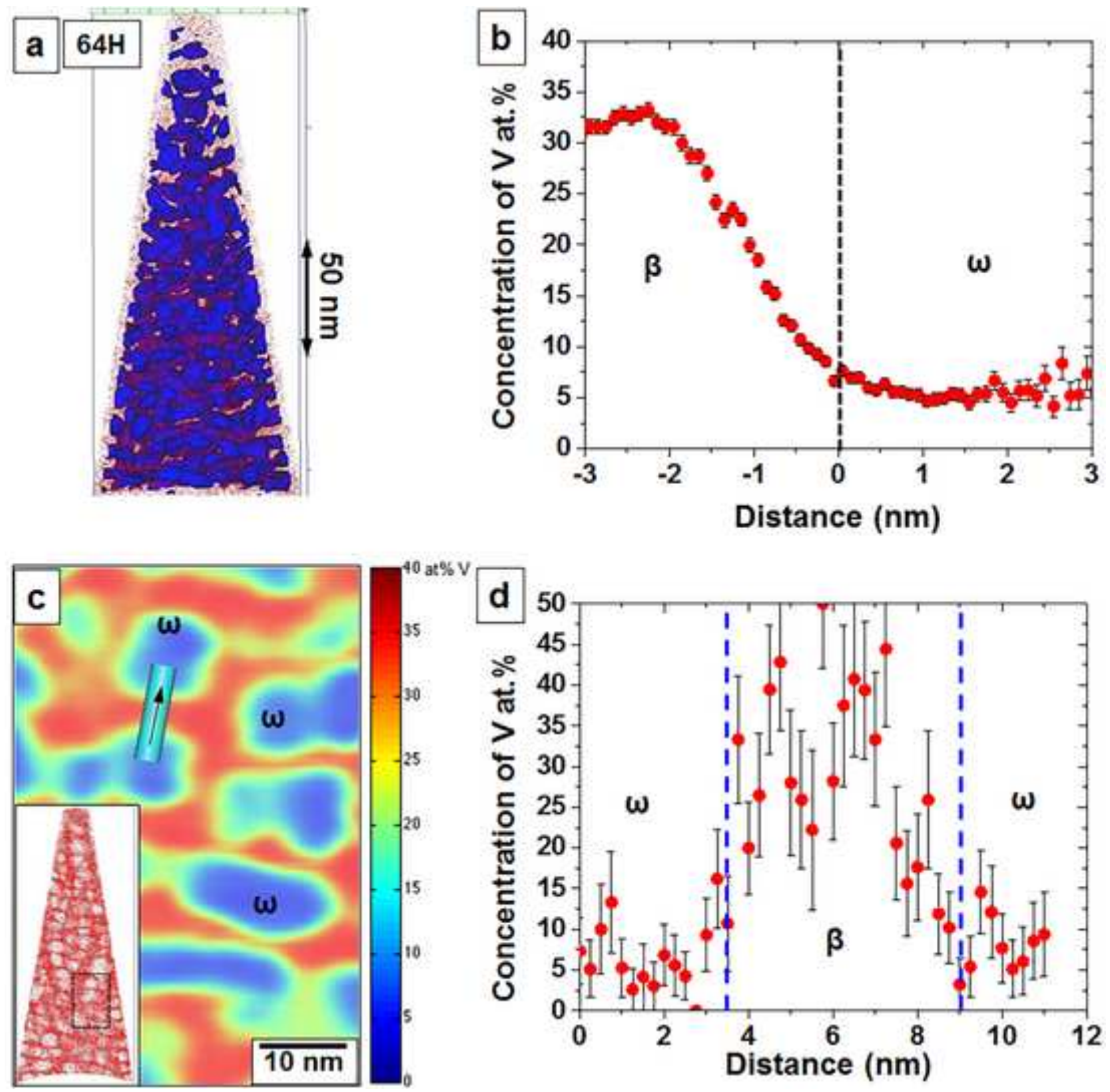

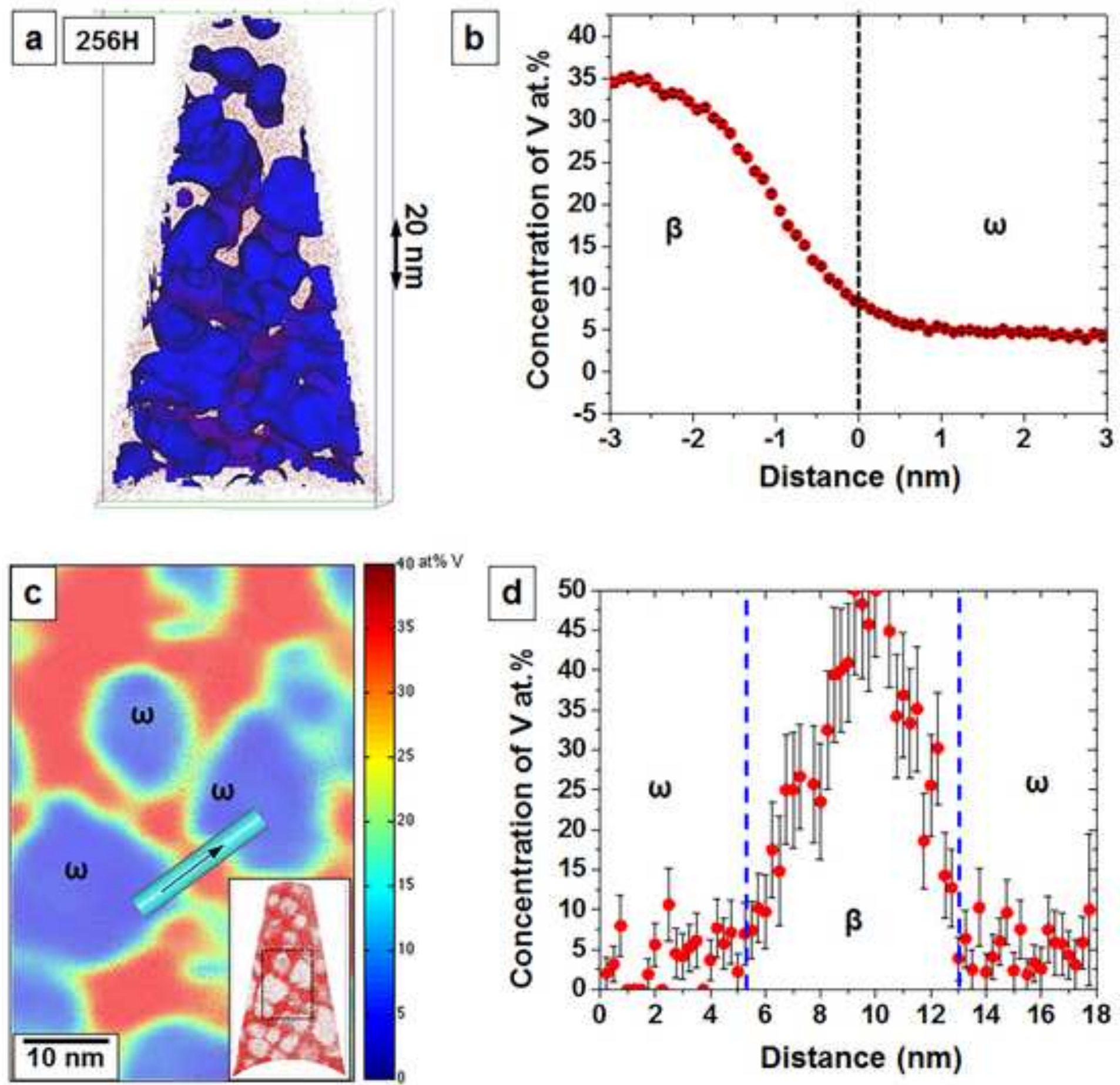
a
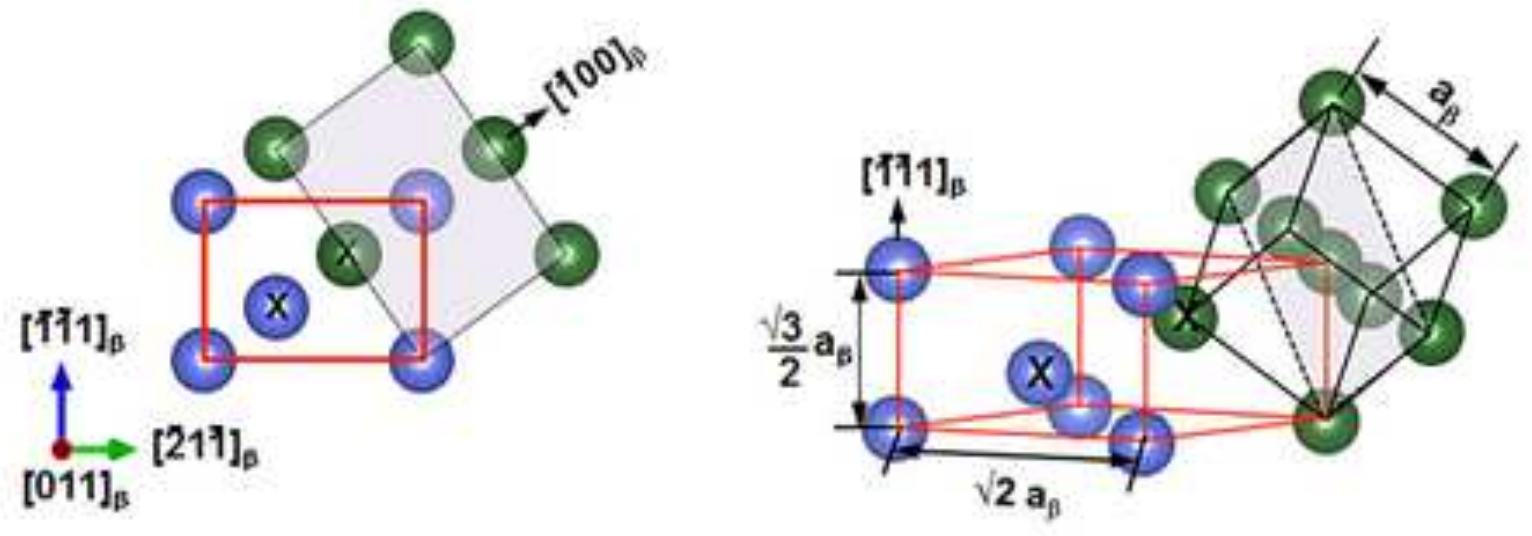

17 at $\% \mathrm{~V}$

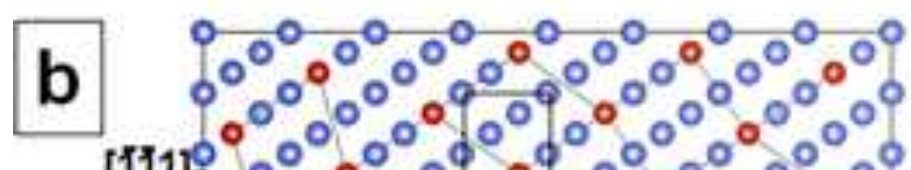
[I11] $000.000,0.000 .000$

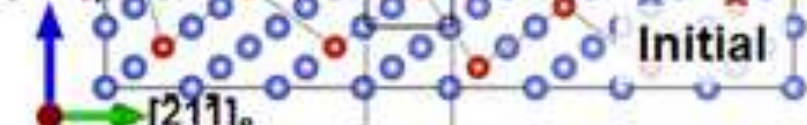

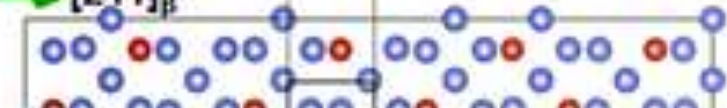

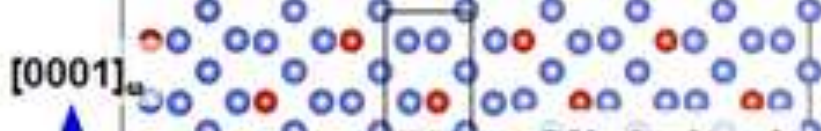

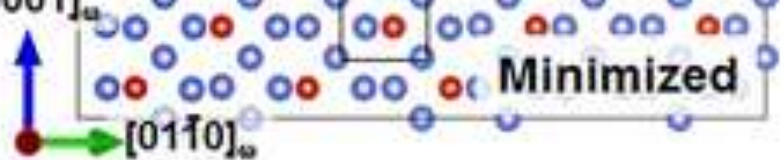
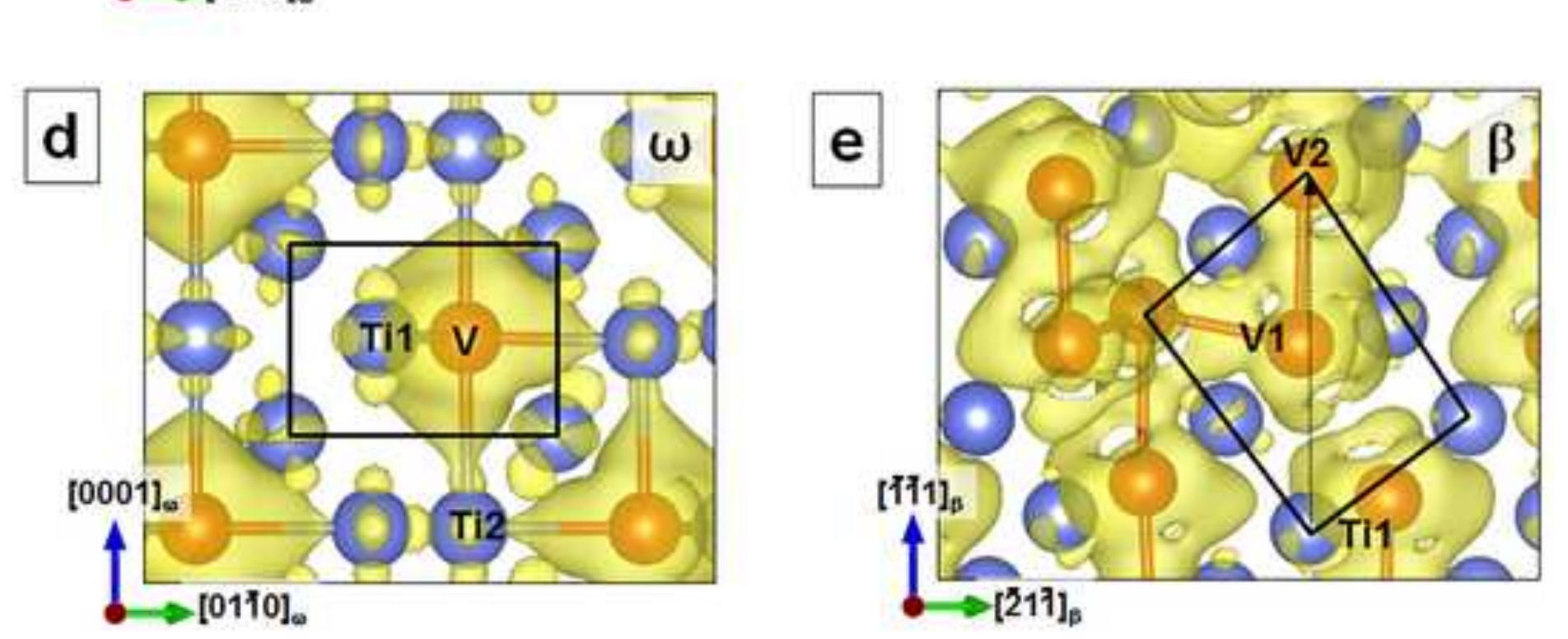

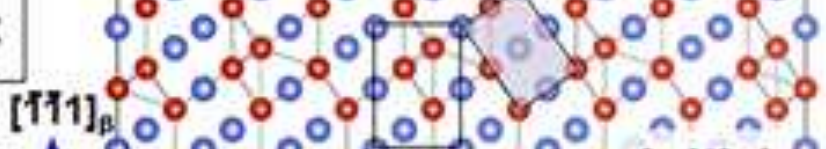
$\int_{0}^{0} 0_{0}^{\circ} 0^{\circ} 0^{\circ} 0^{\circ} 0^{\circ} 0^{\circ} 0^{\circ}$ Initial $\rightarrow[211]_{\beta}$ $0_{0}^{\circ} 0^{\circ} 0^{\circ} 0^{\circ} 9^{2} 0^{\circ} 0^{\circ} 0^{\circ}$

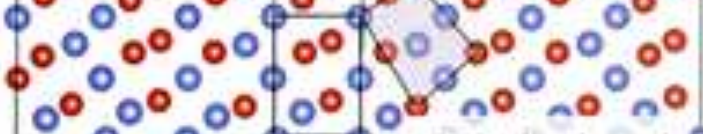
$0.00^{\circ} 0^{\circ} 0^{\circ} \%$ Minimized

\section{6 at $\% \mathrm{~V}$}

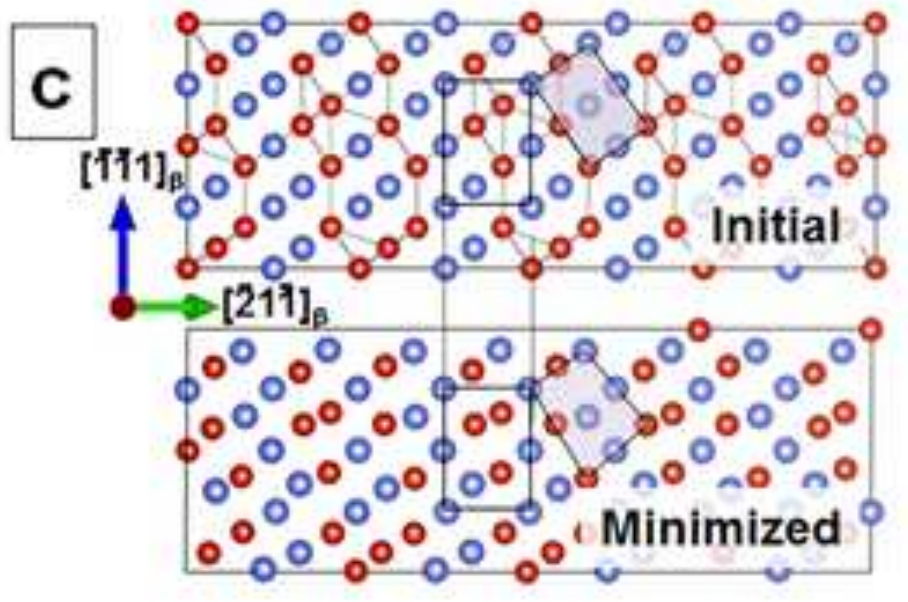

Figure-7

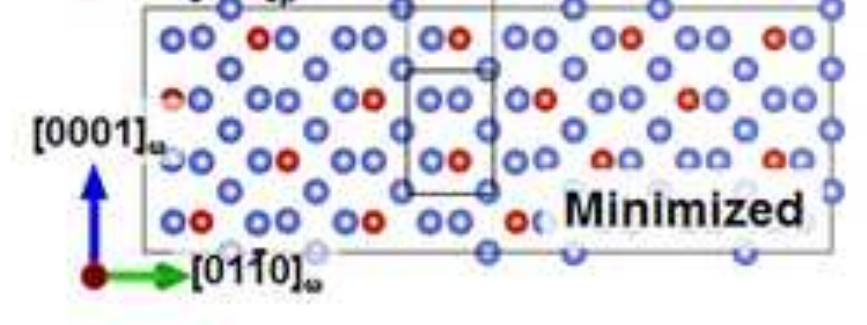




\section{Water-Quenched}

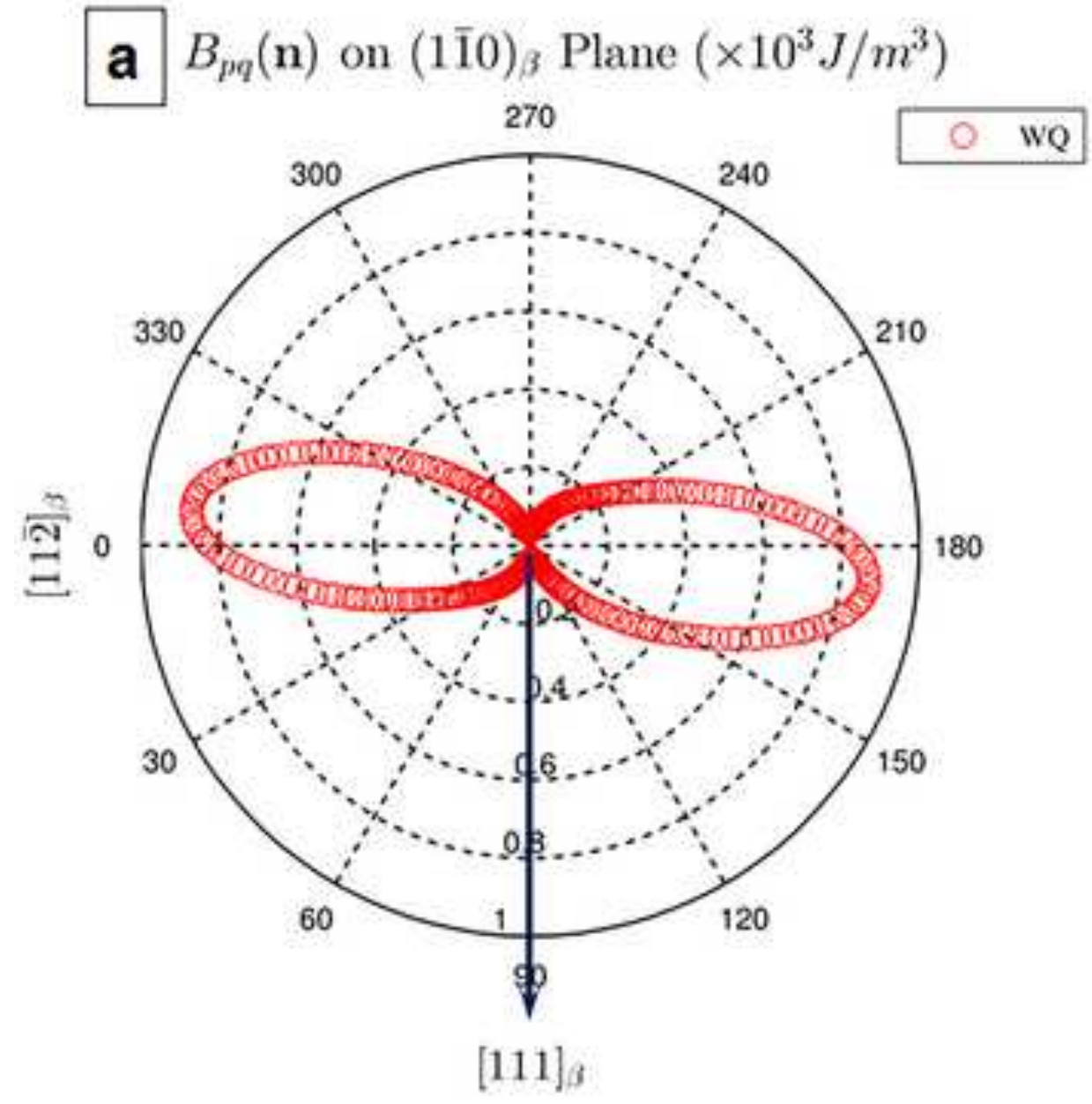

\section{Annealing at $300^{\circ} \mathrm{C}$}

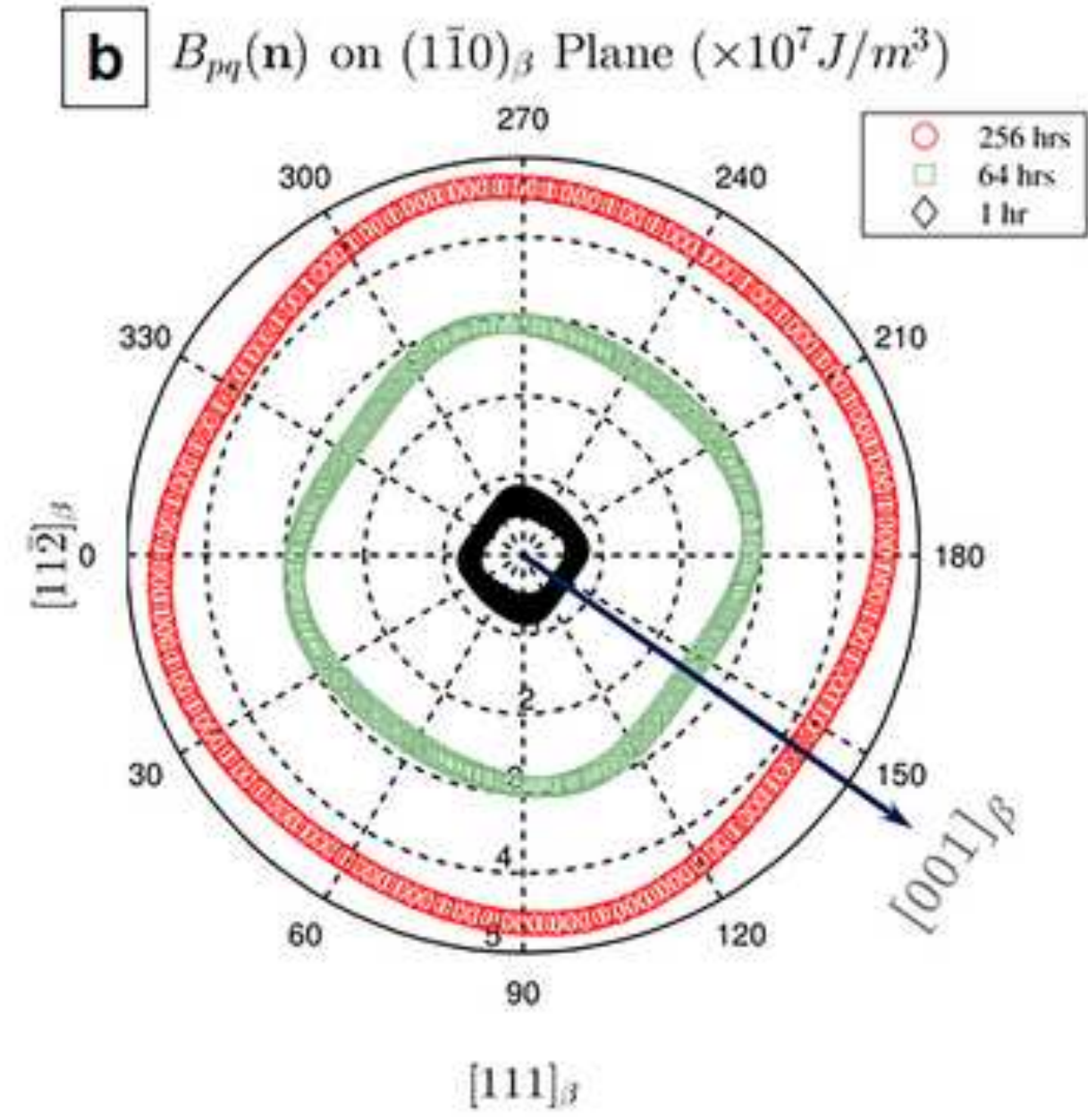




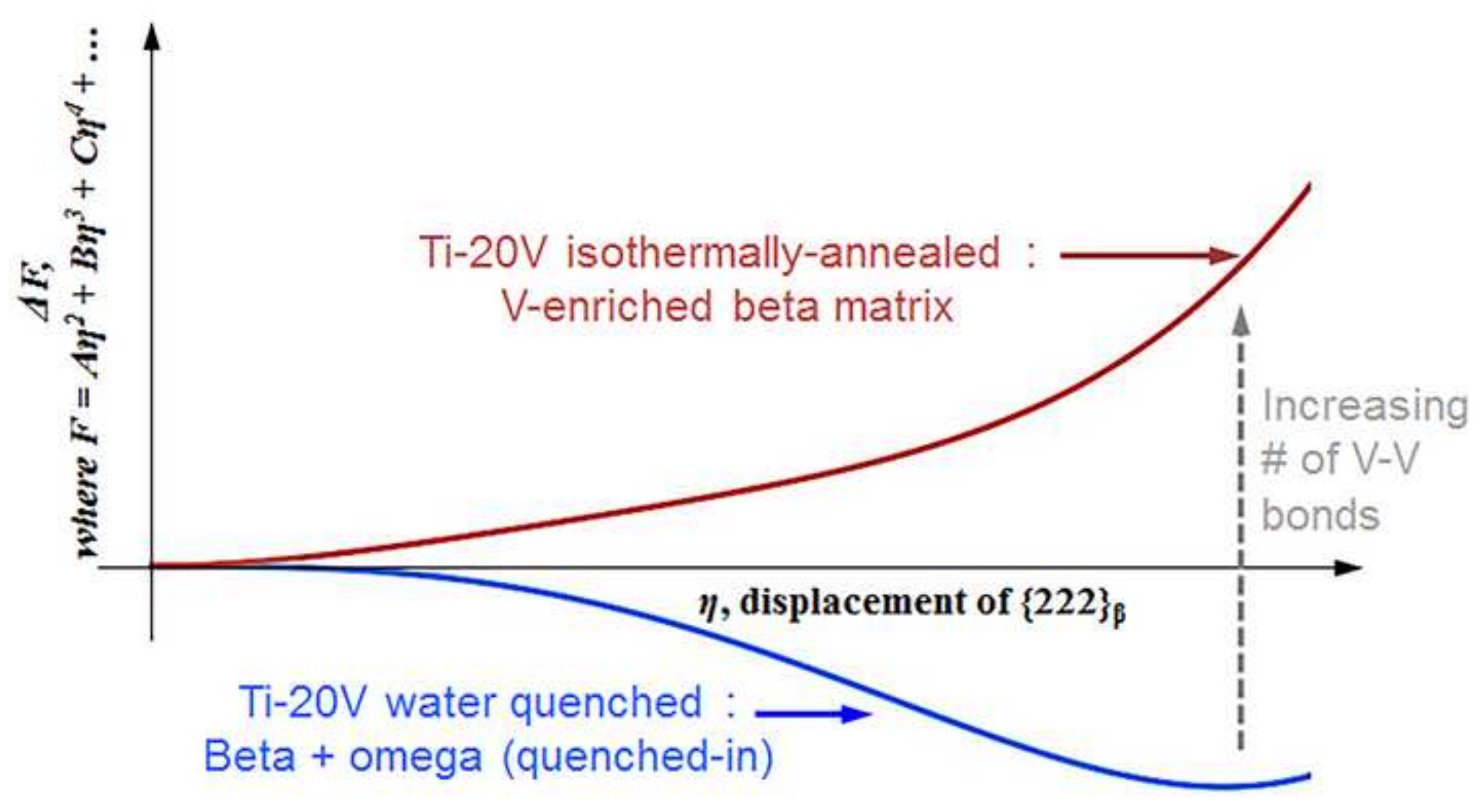

